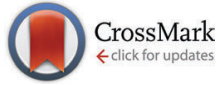

Cite this: Phys. Chem. Chem. Phys., 2014, 16, 24621

Received 9th September 2014, Accepted 3rd October 2014

DOI: $10.1039 / \mathrm{c} 4 \mathrm{cp} 04034 a$

www.rsc.org/pccp

\title{
Chemical imaging of live fibroblasts by SERS effective nanofilm $\dagger$
}

\author{
D. Radziuk, ${ }^{\star a}$ R. Schuetz, ${ }^{b}$ A. Masic ${ }^{b}$ and H. Moehwald ${ }^{a}$
}

\begin{abstract}
Reliable and strong surface enhanced Raman scattering (SERS) signatures of intracellular compartments in live NIH3T3 fibroblasts are collected in real time by means of SERS active thin nanofilm (30 nm) on colloidal silica $(1.5 \mu \mathrm{m})$. Nanofilm is composed of preformed silver nanoparticles in the matrix of polyacrylic acid, protecting against heating $\left(37^{\circ} \mathrm{C}\right)$ in water, or culture medium or phosphate buffered saline aqueous solution. The SERS enhancement factors (EFs) of the order $10^{8}$ allow single biomolecule detection in the native environment of a single live cell. Primary and secondary SERS hot spots of nanofilm are responsible for such high EFs. A slow SERS EF intensity decay occurs over a broader distance of micron silica with nanofilm, not achievable in a common core-shell model (silver nanoparticle coated with a thin silica layer). Extensive local field EFs and SERS EFs are mainly delivered by prolate silver nanoparticles ("rugby-like" shape). This is achieved if an incident field is polarized along the $z$-axis and the direction of incident polarization and main axis $(z)$ are perpendicular to each other, not observable in water or on gold.
\end{abstract}

\section{Introduction}

Investigating proteins and other biomolecules in a living cell environment is a major goal of molecular biology, which has been achieved to a very limited extent so far. Traditional methods for intracellular studies are indirect and require millions of cells to extract specific biomolecules or organelles. ${ }^{1}$ However, the behavior of a single cell varies even in a small population and the true cell metabolism might be obscured. ${ }^{2}$ Moreover, the extraction can cause significant artifacts in the final data. ${ }^{3}$ Therefore there is a need in single-cell techniques to characterize the organelles preserved in their native environment.

A palette of spectroscopy and imaging techniques to study single cells, presumably developed for the early detection of diseases and drug delivery promotion, relies mostly on fluorescence-based methods. ${ }^{4,5}$ Nondestructive analysis with high lateral image resolution enables the morphological study of individual organelles in their native state. However, the need of a fluorescent dye is still a problem for time-dependent studies because of toxicity. ${ }^{6}$ In addition, nonspecific binding can cause a strong background signal lowering the detection sensitivity. Moreover, fluorescence methods are sensitive to quenching processes distorting quantification.

\footnotetext{
${ }^{a}$ Department of Interfaces, Max-Planck Institute of Colloids and Interfaces, D14476 Potsdam, Germany. E-mail: darya.radziuk@mpikg.mpg.de; Fax: +49-331-567-9202; Tel: +49-331-567-9235

${ }^{b}$ Department of Biomaterials, Max-Planck Institute of Colloids and Interfaces, D14476 Potsdam, Germany

$\dagger$ Electronic supplementary information (ESI) available. See DOI: 10.1039/ с4ср04034a
}

Distinct from fluorescence, Raman imaging is a vibrational technique that is based on scattering excluding the necessity of dyes, the problem of toxicity and photobleaching. ${ }^{7}$ Raman vibrational spectroscopy is a powerful diagnostic tool that provides characteristic molecular signatures non-invasively and in a straightforward manner. ${ }^{8}$ Its sensitivity allows single biomolecule detection and diagnostics of structural conformation in native biological environment. However, the intrinsically low cross section results in weak Raman signals $\left(10^{-30}\right.$ and $10^{-25} \mathrm{~cm}^{2}$ compared to fluorescence with $10^{-17}-10^{-16} \mathrm{~cm}^{2}$ ), and the reproducibility is low. Since long it is known that Raman scattering can be strikingly increased at a metallic surface (surface enhanced Raman scattering, SERS) or in the vicinity of plasmonic (e.g. Au or Ag) nanostructures that enable a local electromagnetic field increase. ${ }^{9-11}$ As a great advantage a molecule can perform more SERS than fluorescence cycles per time interval due to the shorter vibrational relaxation times and the molecular prints that can be collected from all molecules in a probed sample even at a minute volume (advantage over IR spectroscopy). On a nano-structured surface single molecule SERS (SM-SERS) arises in the gaps of metallic nanoparticles with the surface plasmon resonance for the local electromagnetic field increase. It usually depends on the nanostructure geometry with the highest values of electromagnetic field at a distance $<10 \mathrm{~nm}$ (SERS hot spots). ${ }^{12,13}$

In a single cell study to make advantage of the SERS hot spots colloidal particles can be adsorbed on a glass nanopipette to increase the Raman scattering cross section of cellular compounds (e.g. DNA, RNA, proteins, and phospholipids), but the orientation of analyte molecules with respect to the SERS 
substrate must be taken into account. ${ }^{14}$ Another strategy towards reliable SERS signals includes the fabrication of core-shell nanostructures bearing a plasmonic-core material and a protective dielectric shell. ${ }^{15}$ However, the narrow choice of composition and breakdown at high or low $\mathrm{pH}$ sufficiently limits its application.

One conceptually simple approach is through chemical binding of the colloids using appropriate surface chemistry. This is however challenging and has not yet been fully explored. As a recent successful example, the nanoshell ${ }^{16}$ approach which is based on gold or silver nanostructures (nanoshell) adsorbed on larger dielectric pearls (silica, polystyrene, etc.) yields more reliable SERS signals. ${ }^{17}$ The term "nanoshell" means a designed shell of metallic nanoparticles bearing plasmonic properties with thickness at nm scale. Such thin plasmonic films can be formed on micrometer substrates of different curvatures which greatly increase the SERS effective area over the entire surface. The recently introduced silver nanofilm can be formed in a matrix of polyethylene glycol (PEG), and the interparticle junctions (SERS hot spots) can be controlled by the polymeric chain length. ${ }^{18}$ Chain-like silver nanostructures with stable and defined interparticle gaps can be prepared in PEG with 8000 Da yielding strong reliable SERS spectra of live fibroblasts in real time. As another advantage, such thin nanofilm can overcome a common problem of dimers: it will become less and less exponentially probable to add an additional particle to the cluster because of the increased barrier height for dimer-sphere interactions and beyond.

Distinct from the core-shell approach, ${ }^{19}$ nanofilms are more flexible and versatile in chemical functionalization and can promote the chemical and physical adsorption of an analyte over a larger SERS effective surface area. As a supporting substrate, silica microparticle, can increase the SERS contact area and nano-roughness of the plasmonic nanofilm allowing SERS detection of proteins, lipids and, due to the broad aromatic ring vibrations of DNA/RNA, of live fibroblasts in real time. ${ }^{20}$ By this approach the problem of uncontrolled silver nanoparticle aggregation can be solved by the protecting polymeric chain groups yielding a SERS effective substrate with geometrically confined stable hot spots. Analytes can be bound to suitable polymers via electrostatic, biospecific or other noncovalent interactions, as the polymers can be coupled in most cases by electrostatic attraction. Design flexibility in the coating density, conformation and porosity of the bound polymer can make advantage at a SERS hot spot with the enhancement values sufficient for single molecule detection. To note, a single polymeric layer can add an additional thickness of less than $1.5 \mathrm{~nm}$ to trap analytes and benefit high electromagnetic field increase. ${ }^{21}$ As another advantage the surface charge density oscillations associated with surface plasmons at the interface between a metal and a dielectric can give rise to strongly enhanced optical near-fields which are spatially confined to the interface, contributing to the SERS enhancement factors. ${ }^{22}$ Moreover, the self-assembly of silica colloids bearing plasmonic nanofilm can provide secondary hot spots in the silicasilica junctions (primary hot spots are formed in the gaps of plasmonic nanoparticles in the nanofilm).
As we have shown above, in SERS a thin plasmonic nanofilm has a number of advantages to chemically image analyte(s) in real time with a relatively low uncertainty. However, the reproducibility of SERS signals (especially of single molecules) is still a problem: probes should have uniformly distributed nanoparticles (uncontrolled aggregation must be avoided), the concentration of plasmonic nanostructures should correlate with the number of analyte(s) and the enhanced SERS signals should be reproducibly strong. As another challenge, most of the cells imaging techniques have to be combined with confocal fluorescence measurements. Based on our previous successful results ${ }^{18}$ we advance a nanofilm approach for SERS studies of single live fibroblasts in real time. We show strong and reproducible SERS signals of single biomolecules in their native environment followed by the label free chemical imaging of the entire cell.

In details the nanoshell approach is advanced by the formation of primary and secondary SERS hot spots consisting of preformed silver nanoparticles in the polymeric matrix of polyacrylic acid (PAA) on colloidal silica, $\mathrm{SiO}_{2} @ A$ A-PAA particles (Scheme 1). Primary hot spots are formed in the gaps of preformed silver nanoparticles $(<10 \mathrm{~nm})$, which are protected by the biocompatible PAA matrix, Ag-PAA (Scheme 1A). Secondary hot spots appear in the silica-silica junctions of the Ag-PAA nanofilm (Scheme 1B). Our approach addresses the problem of low laser intensity for SERS spectra collection and opens the way to a coupled SERS spectral knowledge and a Raman imaging analysis. We also explain how the SERS signals can be intensified by theoretical calculation of the local field intensity enhancement factors (LFI EFs) and SERS EFs of Ag-PAA nanofilm on silica. These findings are thoroughly compared with Ag-PAA in water or with gold $(\mathrm{Au})$ instead of silver. Moreover, we demonstrate how Ag-PAA nanofilm on colloidal silica exhibit the superior advantage of SERS EFs decays versus distance and molecular coverage over a common SERS active "core-shell" model (plasmonic nanoparticles coated with a thin outer layer of silica).
(A)

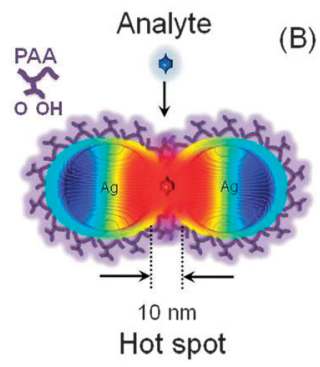

(B)

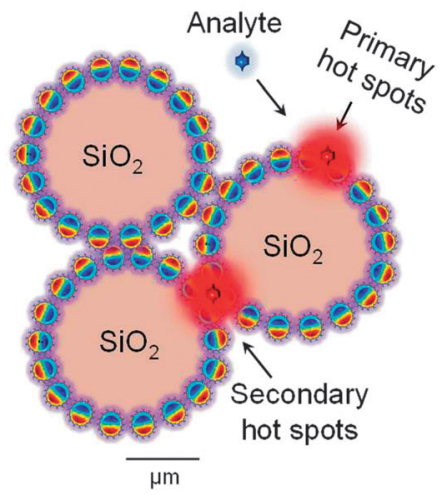

Scheme 1 Schematic illustration of primary and secondary SERS hot spot formation by Ag-PAA nanofilms on colloidal silica. (A) Pre-formed silver nanoparticles are embedded in the matrix of polyacrylic acid (Ag-PAA nanofilms) with fixed gaps $(\sim 10 \mathrm{~nm})$ that can serve as primary SERS hot spots. (B) Ag-PAA nanofilms are deposited on silica microparticles $(\sim 1.5 \mu \mathrm{m})$ that can form secondary SERS hot spots at the interparticle junctions during self-assembly. 


\section{Results and discussion}

\section{Formation of Ag-PAA nanofilm on silica microparticles}

To prepare monodisperse spherical silver nanoparticles (NPs) $(25 \pm 5 \mathrm{~nm})$ we modified the well-known procedure of reduction of silver nitrate with trisodium citrate dihydrate ${ }^{23}$ (Fig. 1A). Silver nanoparticles have a spherical and oval shape with either prolate or oblate spheroid surface (indicated by white arrows in Fig. 1A). The $\zeta$-potential of freshly formed silver NPs was negative $-35 \pm 5 \mathrm{mV}$ and no sediment was observed within a month. As the next step these silver NPs were used for a triple stepwise adsorption on hydrophilic silica microparticles (MPs) $(1.55 \mu \mathrm{m} \pm 0.04 \mu \mathrm{m})$ with terminal Si-OH bonds. Prior to silver NPs deposition the initially negative $\zeta$-potential of silica MPs $(-22.4 \pm 5 \mathrm{mV})$ was reversed to a positive $+44 \pm 5 \mathrm{mV}$ value by functionalizing the hydroxyl groups with aminosilanes ${ }^{24,25}$ (Fig. 1B). After silver NP adsorption the $\zeta$-potential of the silica MPs was reversed to $-45 \pm 5 \mathrm{mV}(\mathrm{pH}=8.5)$. Silica MPs were nonuniformly coated with aggregated silver NPs as isolated islands (average diameter $89 \pm 3 \mathrm{~nm}$ ) (Fig. 1C and Fig. S1A, ESI†).

To avoid aggregation during deposition on silica MPs, silver NPs were incubated in the matrix of polyacrylic acid (Ag-PAA NPs). The stability of nanoparticles was tested during heating at $37^{\circ} \mathrm{C}$ in water (Fig. S2, ESI $\dagger$ ). After heat treatment the negative $\zeta$-potential value of Ag-PAA NPs did not change and no sediment appeared on the bottom within several months which is distinct from bare silver-citrate NPs formed by a Meisel's procedure (Fig. S3, $\mathrm{ESI}+$ ). The UV-Vis absorbance spectrum of Ag-PAA NPs on silica MPs shows a strong band with a slightly red-shifted peak (405 nm) (Fig. 2A). For comparison bare Ag-citrate NPs that are formed by a Meisel's procedure ${ }^{23}$ or modified Xia's method ${ }^{26}$ exhibit absorbance as broad bands with twice or lower intensity (Fig. 2A). Distinct from bare Ag-citrate NPs, silica MPs acquired more uniform coverage by Ag-PAA NPs (Fig. 2B and Fig. S1B, ESI†).

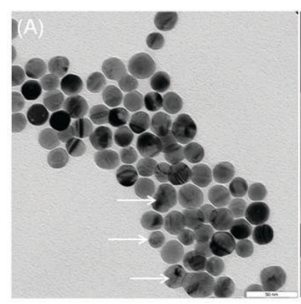

Ag Nanoparticles

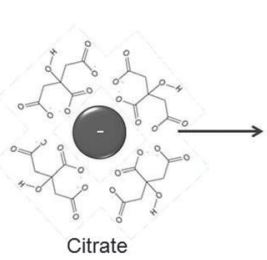

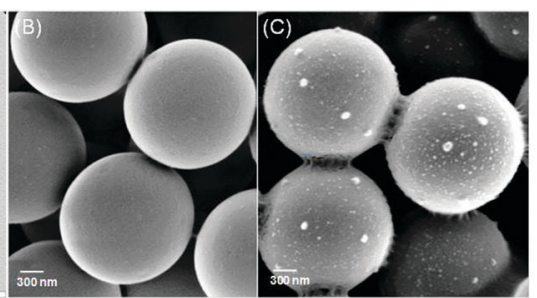

$\mathrm{SiO}_{2}$ Particles
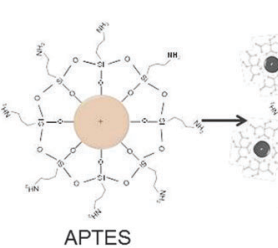

$\mathrm{SiO}_{2}$-Ag Particles

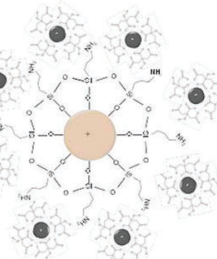

Fig. 1 (A) TEM image of silver-citrate nanoparticles prepared after reduction of silver nitrate with trisodium citrate dihydrate in water (scale bar is $50 \mathrm{~nm}$ ). (B) and (C) SEM images of colloidal silica before and after adsorption of silver-citrate nanoparticles (scale bar is $300 \mathrm{~nm}$ ). The sketch below shows the chemical structure of particles in $A, B$ and $C$.

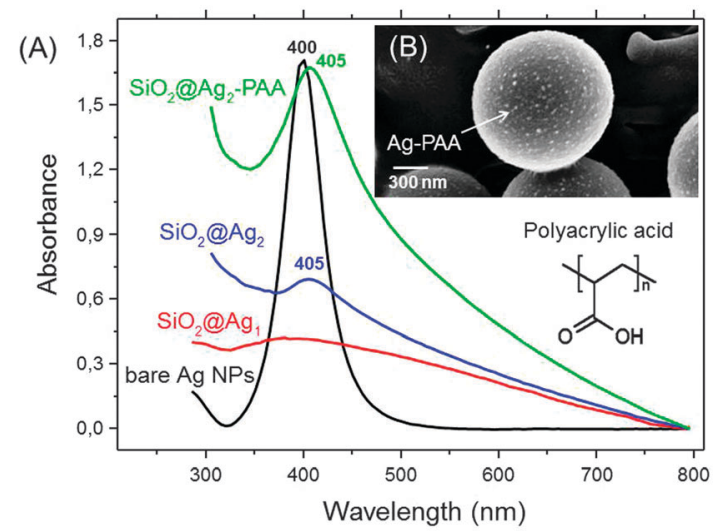

Fig. 2 (A) UV-Vis absorbance spectra of silver nanoparticles (Ag NPs) before and after adsorption on colloidal silica (silica MPs) in water. $\mathrm{SiO}_{2} \mathrm{aAg}_{1}$ (red) and $\mathrm{SiO}_{2} \mathrm{aAg}_{2}$ (blue) indicate that $\mathrm{Ag}$ NPs are formed according to the Meisel's (red) and modified Xia's (blue) procedures followed by their adsorption on silica MPs. $\mathrm{SiO}_{2} \mathrm{aAg}_{2}-\mathrm{PAA}$ (green) indicates $\mathrm{Ag}$ nanofilms in the polyacrylic acid matrix (Ag-PAA) after deposition on silica MPs. (B) SEM image of $\mathrm{SiO}_{2} \mathrm{aAg}_{2}-\mathrm{PAA}$ (green) with the scale bar $300 \mathrm{~nm}$.

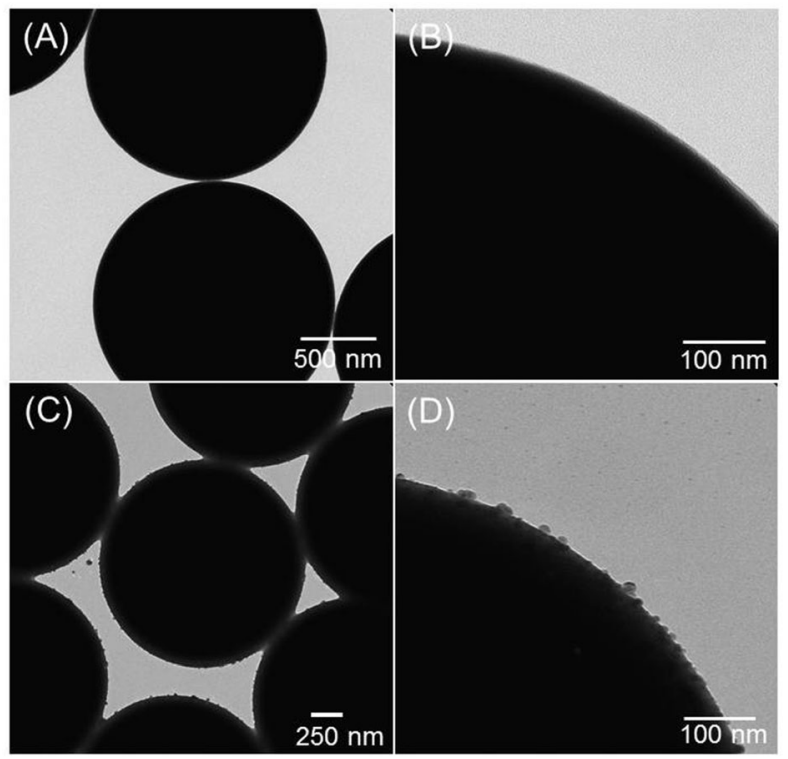

Fig. 3 TEM images of positively charged hydrophilic silica microparticles (silica MPs) (average diameter $=1.5 \mu \mathrm{m}$ ) ( $\mathrm{A}$ and $\mathrm{B}$ ) before and $(\mathrm{C}$ and $\mathrm{D})$ after adsorption of $\mathrm{Ag}$ nanofilms. The scale bars are $500 \mathrm{~nm}(\mathrm{~A}), 100 \mathrm{~nm}(\mathrm{~B})$, $250 \mathrm{~nm}$ (C) and $100 \mathrm{~nm}$ (D).

Before adsorption of Ag-PAA NPs, the positive surface of silica MPs appeared smooth (Fig. 3A and B). After deposition of Ag-PAA NPs the contact surface area between neighbouring silica MPs was increased fourfold with the formation of sharp plasmonic edges in between (Fig. 3C). Fig. 3D shows a TEM image of an individual silica MP with a non-uniform thin layer of Ag-PAA nanofilm, no agglomerates were observed.

As the next step described below, $\mathrm{SiO}_{2} @ A g-\mathrm{PAA}$ particles were examined for the SERS efficiency of rhodamine 6G (Rh6G) molecules in aqueous solution. 


\section{SERS efficiency of rhodamine 6G on Ag-PAA nanofilm}

To test the SERS efficiency, $\mathrm{SiO}_{2} @ A g-P A A$ particles were incubated in the presence of Rh6G with $10^{-4} \mathrm{~mol} \mathrm{~L}^{-1} \mathrm{NaCl}$. From our previous SERS measurements of Rh6G on silver NPs: ${ }^{18}$ it takes less than seconds for SERS spectra detection. However, an incubation time of at least tens of minutes is necessary to achieve a thermodynamic equilibrium between a dye molecule and a silver nanofilm on silica MPs. To note, an incubation period of aqueous solution with nanoparticles can be longer (up to several hours). However, in medical diagnostics in a flow rate detection system, where a SERS effective substrate (not a bulk colloidal solution) can be fixed, no incubation time is required.

The presence of $\mathrm{Cl}^{-}$anions is necessary in order to assist specific chemisorption of dye molecules on Ag NPs via a N-Ag covalent bond. ${ }^{27}$ Rh6G aqueous solution at $10^{-8}$ mol L ${ }^{-1}$ concentration was sufficient for low threshold surface enhancement $\left(10^{6}\right)$, which is accompanied by suppression of fluorescence ${ }^{28}$ (Fig. S4, $\mathrm{ESI} \dagger$ ). The effective number of $\mathrm{Rh} 6 \mathrm{G}$ on the $\mathrm{SiO}_{2}$ @Ag-PAA surface is about 14 dye molecules per silver NP, assuming the surface areas of silica and silver as $1.89 \mu \mathrm{m}^{2}$ and $3.14 \times 10^{-4} \mu \mathrm{m}^{2}$ (more details can be found in the Experimental section).

The SERS spectra of Rh6G were collected on single $\mathrm{SiO}_{2} @ A g-P A A$ particles, their dimers or closely packed selfassembly consisting of four-five particles (Fig. 4A), that are designated as A, B or C, respectively (Fig. 4B). The SERS spectra are reproducible and cumulatively averaged over at least ten particles of each type (Fig. S5, ESI $\dagger$ ). Overall the intensity of SERS bands was increased by two orders of magnitude during the self-assembly of particles due to the increased number of interparticle junctions. The estimated SERS EFs of Rh6G on the closely packed $\mathrm{SiO}_{2} @ A g-P A A$ particles are of the order of $10^{8}$ being sufficient for single molecule detection (details can be found in the Experimental section).

The density of Ag NPs is higher on C than on A or B as silverhalide vibration $\left(246 \mathrm{~cm}^{-1}\right)$ appears with increased broadening and smoothing $\left(\mathrm{Cl}^{-}\right.$anions are forced to desorb) followed by a shift to a higher frequency ${ }^{30}$ (Fig. 4A).

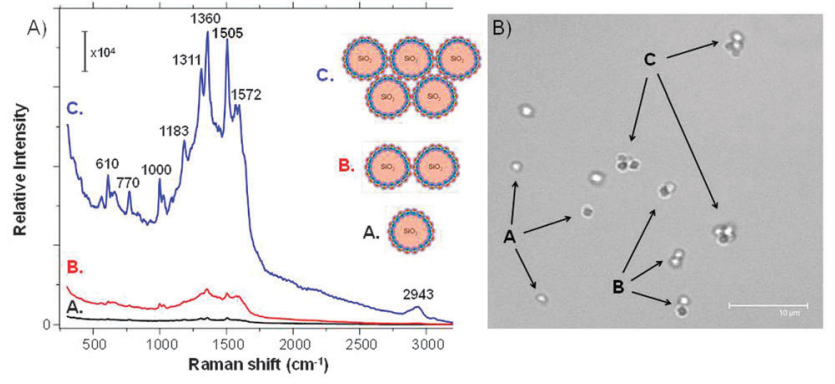

Fig. 4 (A) Surface enhanced Raman scattering (SERS) spectra of Rh6G $\left(10^{-8} \mathrm{~mol} \mathrm{~L}^{-1}\right)$ in aqueous solution of $\mathrm{SiO}_{2}(\mathrm{AAg}-\mathrm{PAA}$ particles as single $(\mathrm{A})$, dimers (B) or closely-packed self-assembly (C). The excitation wavelength was $532 \mathrm{~nm}$ with the grating $600 \mathrm{~g} \mathrm{~mm}^{-1}(B L Z=500 \mathrm{~nm}$ ) and a spectral resolution of $3 \mathrm{~cm}^{-1}$. The SERS spectra are cumulative and averaged over at least ten particles of each type. (B) Confocal laser scanning microscopy (CLSM) image of these particles in a bulk aqueous solution (scale bar is $10 \mu \mathrm{m}$ ).
The conformational distortion of the hydrogen atoms of the xanthene skeleton (at $610 \mathrm{~cm}^{-1}$ and $770 \mathrm{~cm}^{-1}$ ) 29 is the strongest on $\mathrm{C}$ due to a weaker chemisorption of the Rh6G dye. To note, the full width at half maximum (FWHM) of this band does not change, as only one vibration contributes to this signal. Strong bands of pyridine $\left(992 \mathrm{~cm}^{-1}\right.$ and $\left.1030 \mathrm{~cm}^{-1}\right)$ are intensified on $\mathrm{C}$, but at shifted frequencies $\left(1000 \mathrm{~cm}^{-1}, 1026 \mathrm{~cm}^{-1}\right.$ and $1086 \mathrm{~cm}^{-1}$ ), not observable in free $\mathrm{Ag}$ NPs. ${ }^{18}$ The absolute intensity of the $\mathrm{C}-\mathrm{H}$ in-plane bending coupled to the $\mathrm{C}-\mathrm{C}$ stretching $\left(1181 \mathrm{~cm}^{-1}\right)^{28}$ is fivefold stronger on $\mathrm{C}\left(\right.$ at $\left.1183 \mathrm{~cm}^{-1}\right)$ as well as the $\mathrm{C}-\mathrm{H}$ stretching at $1311 \mathrm{~cm}^{-1}$ and $1360 \mathrm{~cm}^{-1}$.

Another $\mathrm{C}-\mathrm{C}$ band $\left(1509 \mathrm{~cm}^{-1}\right)$ appears at $1505 \mathrm{~cm}^{-1}$ as a strong band on $\mathrm{C}$, while the aromatic ring of this vibration is split into a doublet $\left(1572 \mathrm{~cm}^{-1}\right.$ and $\left.1595 \mathrm{~cm}^{-1}\right)$ and the band at $1650 \mathrm{~cm}^{-1}$ gradually disappears. It is known, that this carbon skeleton band is extremely sensitive to molecular distortion, i.e. coupling between the $\mathrm{Cl}^{-}$and silver-Rh6G complexes. This sensitivity is enhanced by two orders of magnitude on $\mathrm{C}$ and is not observable in free Ag NPs. ${ }^{18}$

However, $\mathrm{SiO}_{2} @ A g-\mathrm{PAA}$ particles do not enhance the in-plane $\mathrm{N}-\mathrm{H}$ deformations at $1240-1250 \mathrm{~cm}^{-1}$ and the pyridinium halide $\mathrm{C}_{5} \mathrm{H}_{5} \mathrm{NH}^{+} \mathrm{Cl}^{-}$vibration $\left(2380 \mathrm{~cm}^{-1}\right)$, similar to free $\mathrm{Ag}$ NPs. ${ }^{18}$ Although the characteristic ring vibrations with considerable $\mathrm{N}-\mathrm{O}$ stretching are not observed at $1252 \mathrm{~cm}^{-1}$ this vibration appeared as small peaks with lower frequency shifts $\left(543 \mathrm{~cm}^{-1}\right.$ and $835 \mathrm{~cm}^{-1}$ ), distinct from free $\mathrm{Ag}$ NPs. To note, the $\mathrm{O}-\mathrm{H}$ vibrations of water $\left(2943 \mathrm{~cm}^{-1}\right)$ that are initiated by the electromagnetic field enhancement are also strongly enhanced on C.

Overall, Ag-PAA nanofilm efficiently enhance Raman signals of Rh6G with the EF $\left(10^{8}\right)$, more than sufficient for single molecule detection. The SERS increase is higher from several self-assembled silica MPs due to the formation of interparticle junctions between Ag-PAA nanofilms, which can act as secondary SERS hot spots. However, it is unclear how such high SERS EFs can be achieved and whether the nanoparticle geometry in the nanofilm can be responsible for the local field intensity EFs. Moreover, it is unknown if there is a dominance in the SERS EF increase of silica as a micron-substrate or as a thin outer layer (typically several nm only). Can silver be substituted by gold in the introduced nanofilm in order to reach such high SERS EFs and what are the possible conditions for this? To start with, we decided to calculate EFs of the local field intensity as well as maximum and average SERS EFs on Ag-PAA nanofilm on silica, in water and compare them with gold and a common core-shell model (silver or gold nanoparticle coated with a thin silica layer).

\section{Theoretical calculation of SERS-EF and LFI EF (local field intensity) enhancement factors of Ag-PAA nanofilm}

In the frame of the generally accepted distance dependence in the electrostatic approximation (ESA) we calculated the cumulated SERS EFs in a $\mathrm{SiO}_{2} @ A g-P A A$ particle (Fig. 5A) and compared it with a core-shell model consisting of Ag NP as a core coated with a thin silica-shell (Fig. 5B) over the distance from the SERS effective surface. In our calculation we restrict ourselves to situations of reasonably large enhancements, where the scattered field dominates over the incident field. 
The electric field outside the particle in the ESA is created by an electrostatic dipole at the center of a particle. For a molecule at a distance $d$ from the particle surface, the electric field amplitude should decay as $1 /(a+d)^{3}$, where " $a$ " is a radius of a particle and " $d$ " is a distance from its surface. Punctual and average SERS EFs should decay as $1 /(a+d) .{ }^{12}$ Despite a dramatic decay due to an exponent as large as 12 a SERS signal can be dominated by more than the first layer of adsorbed molecules because $a \gg d$ in both examples of particles.

Although the SERS EF is maximum at $d=0$ (first layer), the EM enhancements are long-range effects and can extend to at least $10 \mathrm{~nm}$ away from the metallic surface. In the frame of the ESA one can directly predict the coverage dependence of the SERS signal (i.e. the number of molecular monolayers on the surface) from the distance dependence of the SERS EF. Because the SERS EF decreases with distance, the cumulated SERS signal varies sub-linearly with coverage and saturates.

In our calculation the SERS-EFs are plotted versus coverage at Ag-PAA nanofilm on silica MPs with their radius varied from $50 \mathrm{~nm}$ to $2000 \mathrm{~nm}$ (Fig. 5A). A monolayer thickness is assumed to be $0.5 \mathrm{~nm}$. Sublinear decay of the SERS EF is slower on $\mathrm{SiO}_{2}$ of micron size (e.g. $1 \mu \mathrm{m}$ or $2 \mu \mathrm{m})$ with saturation approaching at far above $10 \mathrm{~nm}$ followed by a larger coverage of analytes experiencing high SERS EF. This means more and different analytes can be potentially detected and examined at a single molecule level in this configuration. This is in contrast with smaller $\mathrm{SiO}_{2}$ particles (i.e. < several hundred $\mathrm{nm}$ ) where the SERS EF saturates faster in the proximity of $10 \mathrm{~nm}$. This means that the challenge of analyte positioning directly on a hot spot (typically $10 \mathrm{~nm}$ distance from the metal surface) can be overcome by using $\mathrm{SiO}_{2}$ particles rather of micron than $\mathrm{nm}$ size. However, for practical purposes many additional effects should be also considered at multilayer coverages. For example, the molecules in the first layer may be chemically influenced by the metallic surface and the adsorption geometry may be different in subsequent layers. To note, the average and maximum SERS $\mathrm{EF}$ is rather a widespread property of the enhancement on the entire surface of our particle(s). This is in agreement with the tendency of higher electromagnetic field increase with the increase surface area of plasmonic nanoparticles. ${ }^{31}$
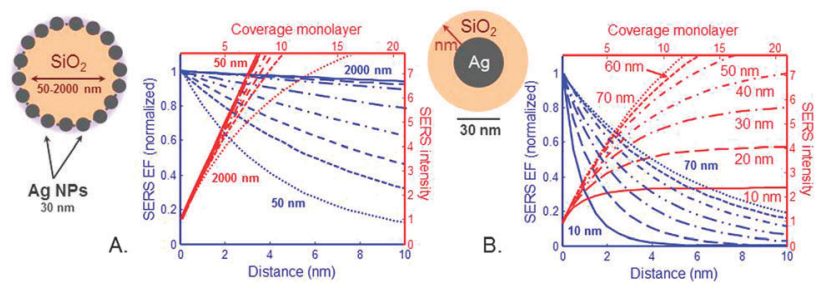

Fig. 5 Dependence of electromagnetic SERS enhancement factors (EFs) with the distance $d$ and coverage. (A) Ag-PAA nanofilm (radius $30 \mathrm{~nm}$ ) on silica MP designated as $\mathrm{SiO}_{2} @ \mathrm{Ag}-\mathrm{PAA}$ particle with a radius of silica MP that is varied from $50 \mathrm{~nm}$ to $2000 \mathrm{~nm}$. (B) Ag NP (radius $30 \mathrm{~nm}$ ) as a core coated by a thin silica outer layer with the thickness from $10 \mathrm{~nm}$ to $70 \mathrm{~nm}$. The cumulated average SERS intensity (on the right axis) is plotted as a function of analyte coverage assuming a monolayer thickness of about $0.5 \mathrm{~nm}$.
To remind, the local surface plasmon (LSP) resonances are mostly radiative (especially dipolar one), resulting in a larger radiative decay rate when the dipole couples to them efficiently. These radiative resonances and the main dipolar LSP resonance are strongly size-dependent for spheres and more generally are geometry-dependent, being largest for smaller spheres (nm range). For comparison we calculated the cumulated SERS EFs versus distance and coverage for a common SERS system: core-shell particle, consisting of Ag NP (30 nm) as a core being coated by a thin outer silica layer. The thickness of the silica coating is varied from $10 \mathrm{~nm}$ to $70 \mathrm{~nm}$ (Fig. 5B). From our calculation the SERS EF intensity saturates fivefold faster at a distance $<2 \mathrm{~nm}$ with a silica shell thickness of $10 \mathrm{~nm}$ and at $\sim 5 \mathrm{~nm}$ with a silica shell thickness of $70 \mathrm{~nm}$. At this distance range the coupling of an analyte to these resonances is not strongly distance-dependent. However, it is sensitive to the dipole orientation: the perpendicular dipole couples more efficiently to the LSP resonances. To note, in this SERS system an analyte should be closely brought to the metallic surface $(d=0-2 \mathrm{~nm})$ or positioned at the interparticle junction in order to benefit higher SERS EFs, which is problematic for single molecule studies. As another fact, the coverage thickness of analytes is several $\mathrm{nm}$ less in this SERS system at a minimum detection distance of about $8 \mathrm{~nm}$, which further lowers the potential ability of this system to overcome problems in single molecule studies.

In the frame of the ESA we also calculated the local field intensity enhancement factors (LFI EFs) of Ag-PAA nanofilm by applying an analytical solution (details in the Experimental section) (Fig. 6). As the shape of Ag NPs in the PAA matrix strongly changes from spherical to ellipsoidal at $37{ }^{\circ} \mathrm{C}$ (conditions for living cell studies) (Fig. S1, ESI $\dagger$ ) the LFI EFs were estimated for prolate or oblate silver spheroids (30 nm) (Fig. 6).

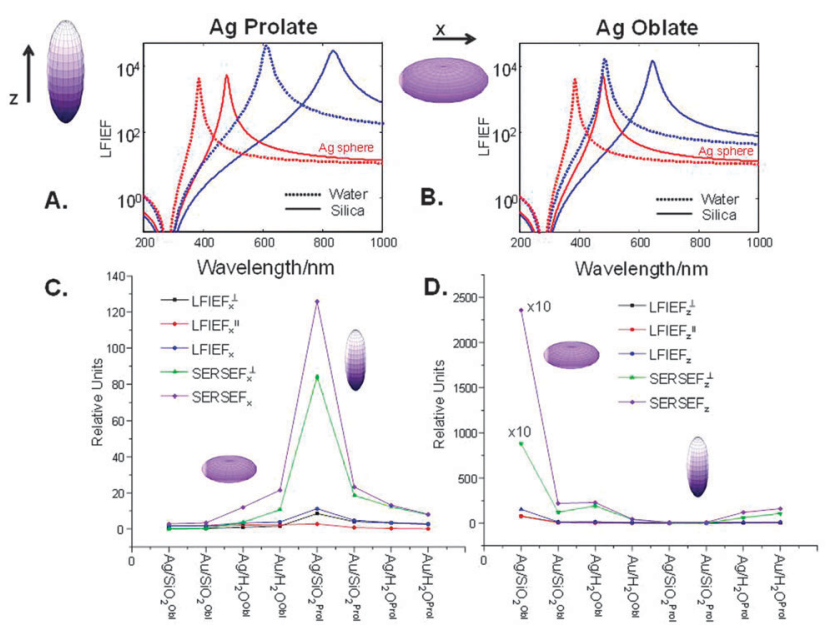

Fig. 6 (A) and (B) Wavelength dependence of the maximum ( $A^{\text {perp }}$ ) and minimum ( $A^{\text {para }}$ ) of the local field intensity enhancement factor (LFI EF) for the prolate and oblate $\mathrm{Ag}$ spheroid with aspect ratio 3 (dashed lines indicate those in water and solid lines - in silica). "Ag sphere" is the corresponding quantity for a sphere given for comparison. (C) and (D) LFI EFs and SERS EFs on $\mathrm{Ag}$ oblate or prolate spheroids in nanofilm on silica or in water in comparison with gold at the incident polarization along $x$ or $z$ axes. 
The calculated LFI EFs of $\mathrm{SiO}_{2} @ A g$-PAA particles were compared with $\mathrm{Ag}$ nano-spheres $(30 \mathrm{~nm})$. As for the sphere we can consider the LFI EF at selected positions on the ellipsoid surface (at the corners along the main axes) at an incident field polarized along one of the axes of the spheroid ( $z$ for prolate or $x$ for oblate). To note, the LFI EFs at all points on the surface can be expressed in terms of the non-dimensional polarizability $\beta_{i}(\omega)$ along the incident polarization axis ( $z$ or $x$ ), distinguishing between local field perpendicular and parallel to the surface. The maximum $\left(A^{\text {perp }}\right)$ and minimum $\left(A^{\text {para }}\right)$ values characterize the maximum and minimum LFI EFs for incident polarization along $e_{i}$. The maximum enhancement, $A^{\text {perp }}$, is obtained at the "tips" of the axis, and the local field is perpendicular to its surface. The minimum enhancement, $A^{\text {para }}$, is obtained at the four points on the two other axes and the local field is tangential to the surface.

In the ESA for LFI EFs we have to distinguish between excitations along a "pointy" or "flat" axis:

- For excitation along axes with "flat" curvatures $\left(L_{i}>1 / 3\right)$, the factor $\left(3 L_{i}\right)^{-2}$ contributes to a decrease of the LFI EF at a resonance compared to the sphere case. This decrease is even larger for $A^{\text {perp }}$ because of the dependence of $\left|\varepsilon\left(\lambda_{i}\right)\right|^{2}$ on $L_{i}$.

- For excitation along axes with "pointy" curvatures $\left(L_{i}<1 / 3\right)$ the factor $\left(3 L_{i}\right)^{-2}$ contributes to an increase of the LFI EF at a resonance compared to the sphere case. This increase is further magnified for $A^{\text {perp }}$ because $\left|\varepsilon\left(\lambda_{i}\right)\right|^{2}$ increases as the resonance wavelength red-shifts (which is the case for decreasing $L_{i}$ ).

Overall, Fig. 6 illustrates the wavelength dependence of the maximum $\left(A^{\text {perp }}\right)$ and minimum $\left(A^{\text {para }}\right)$ LFI EFs of prolate or oblate spheroids with aspect ratio 3 (blue curves) in comparison with a sphere ( $\mathrm{Ag}_{\text {sphere }}$, red curves) in silica (solid lines) or water (dashed lines). The LFI EFs are higher for Ag prolate spheroids with a strong red-shift near $850 \mathrm{~nm}$ (Fig. 6A), but being close to $650 \mathrm{~nm}$ for $\mathrm{Ag}$ oblate spheroids (Fig. 6B). This effect is more pronounced on silica than in water due to the higher value of the dielectric constant. It is important to emphasize, that LFI EFs are of the order between $10^{4}$ and $10^{5}$ (corresponding to the SERS EF between $10^{8}$ and $10^{10}$ ), in agreement with the experimental data and potential ability for meaningful single molecule studies. In contrast, LFI EFs of bulk $\mathrm{Ag}_{\text {sphere }}$ are slightly below $10^{4}$ on silica or in water (Table S1, ESI $\dagger$ ).

High enhancement values are observed in the Ag-silica system when the directions of incident polarization and main axis of a spheroid are perpendicular to each other, being several times larger than in the Ag-water or $\mathrm{Au}$-water systems. To note, the LFI EFs in Au spheroids are much smaller with a resonance that is strongly damped, because it occurs at wavelengths where the optical absorption is large. However, for prolate Au spheroids these values are slightly larger as the red-shifted resonance is no longer affected by this problem (Table S1, ESI $\dagger$ ). This resonance should have comparable properties as that of silver ellipsoid. This means that SERS studies of live cells can be potentially improved by minimizing drawbacks of particle toxicity, increasing biocompatibility and broadening the surface functionality of a potential SERS effective platform based on the nanofilm approach.

Moreover, prolate spheroids (Ag or $\mathrm{Au}$ ) with a "rugby-like" shape exhibit larger maximum EFs than oblate spheroids ("pumpkin-like" shape) of both metals and can reach the SERS EF of $10^{10}$ (LFI EF $\sim 10^{5}$ ) more than sufficient for single molecule studies. In this way, these results illustrate an important point, often the source of confusion: in SERS gold substrates can be as efficient as those of silver when resonances are sufficiently red-shifted (i.e. Au prolate spheroids with the aspect ratio $\geq 3$ and those of $\mathrm{Ag}$ being sufficient with $\geq 2$ ). In this way, a nanofilm approach, based on the stability of preformed $\mathrm{Ag}$ or Au nano-geometries in the polymeric matrix of a specific interest, can offer a great variety of functional hot spots, hence reliable LFI EFs to perform single molecule studies.

From the above arguments that large additional enhancements can be achieved at specific points of prolate spheroids, let us consider also the average SERS EFs on these particles when analytes are randomly adsorbed over the surface (Fig. 6C and D). General expressions are described in details in the Experimental section. To note, the average SERS-EFs can be higher by two orders of magnitude for Ag-silica oblate spheroids, when the incident polarization is along $z$ direction. However, it can be about ten times larger for $\mathrm{Ag}$-silica prolate spheroids, when the incident polarization is along $x$ direction, in agreement with the theoretical predictions elsewhere. ${ }^{32}$ This is true, bearing in mind the same aspect ratio, that minimum average SERS-EFs can be observed for both Ag-silica oblate or prolate spheroids, when the incident polarization coincides with their main axes. In the latter case, minimum enhancement values for silver are comparable with those for gold. However, as a result of a "localization" effect the maximum SERS EF can be also increased, but applied to a smaller proportion of the surface area (oblate shape). Upon surface averaging the average SERS EF can be increased, but partly compensated by localization, hence the importance of hot spots (interparticle junctions) is critical.

From calculation above we found out that the proposed Ag-PAA nanofilm approach offers a number of benefits for not only higher SERS EFs, but also LFI EFs. These values are several fold higher on silica as a micron substrate than as a coating shell of $\mathrm{nm}$ thickness. Considering the lower aspect ratio, Ag-PAA NPs with prolate shape exhibit stronger EFs (up to $10^{10}$ ) on silica MPs than a similar system in water or with gold. However, at the equivalent higher aspect ratio such high EFs can be achieved also with gold instead of silver, which are important for live cellular studies. Lower EFs $\left(10^{6}-10^{8}\right)$, however sufficient for single molecule detection, can be provided by oblate silver or gold nanoshells in a comparable fashion. As we have demonstrated above (experimentally and theoretically) Ag-PAA nanofilm can be used as SERS efficient platforms to intensify weak Raman signals of biomolecules from a live cell in real time. Let us further demonstrate how Ag-PAA nanofilm can effectively increase molecular prints of biomolecules of a single live fibroblast in real time.

\section{Chemical imaging of live fibroblasts by Ag-PAA nanofilm}

$\mathrm{SiO}_{2}$ @Ag-PAA particles were embedded into the interior of live NIH/3T3 fibroblasts by electro-permeabilization ${ }^{33}$ of the cell membrane. The optical phase contrast images of live fibroblasts before (Fig. S6A, ESI $\dagger$ ) and after (Fig. S6B, ESI $\dagger$ ) electroporation show no visible changes of the cell damage or 
apoptosis. In general, in all experiments the cell viability after electroporation was optically monitored and compared with control cells free of particles. For comparison SERS effective nanofilm was prepared also with gold nanoparticles instead of silver.

After electroporation we observed fibroblasts, which preserved their native morphology (designated as "normal"), those with the acquired round shape due to the reduced adhesion affinity (designated as "round") and those that acquired an increased volume of a vacuole (designated as "vacuole"). To remind, a vacuole changes its structure to the needs of the cell, e.g. when it isolates materials that might be harmful or a threat to the cell, among other functions.

Fig. S7A (ESI $\dagger$ ) shows the statistics of live fibroblasts with normal or round morphology followed by those with increased number of visible vacuoles after electroporation. Overall, the majority of cells were observed as normal (about 75-95\%) with almost no difference between silver or gold nanofilms. Less than $20 \%$ of the cells acquired round shape and vacuoles with increased volume. The number of cells with embedded silver nanoshells is about the same in both types, but it is different with gold nanofilms: the number of round cells is slightly higher and fewer those with vacuoles. This means that only one fifths of all the cells experience reasonable changes of the physiological conditions in order to maintain the main cellular functions. To note, similar changes after electroporation were observed with the control cells free of particles. It is important to emphasize, that for a development of a non-destructive labelfree chemical imaging electroporation should be replaced by alternative non-damaging methods.

In addition, the applied voltage and the duration of electroporation were varied in order to find out the appropriate conditions at which the number of normal cells can be higher. The statistics of live cells after different types of experiment is plotted in Fig. S7B (ESI $\dagger$ ). At applied voltage below a certain minimum threshold value (i.e. $\pm 200 \mathrm{~V}$ ) during $750 \mathrm{~ms}$ the effect is comparable to incubation/aging. However, above $\pm 500 \mathrm{~V}$ in $350 \mathrm{~ms}$ most of the cells were damaged (independently on the number of $\mathrm{SiO}_{2} @ A g-\mathrm{PAA}$ particles). The majority of normal cells (about 95\%) were observed without any visible morphological changes comparable to the control cells before electroporation (types of experiment \# 9 and 11). As shown later these conditions were used as default set of parameters for electroporation of $\mathrm{SiO}_{2} @ A g-P A A$ particles (Fig. 7 and 8).

For SERS measurements the culture medium was removed and the petri dish with incubated live fibroblasts was triply rinsed with phosphate buffered saline (PBS) solution. As one of the examples, most of the SERS active particles are closelypacked in the area close to or surrounding the nucleus of live fibroblasts (Fig. S8, ESI $\dagger$ ). The SERS effective Ag-PAA nanofilm appear as bright scattering spots of relatively high intensity as indicated by arrows (Fig. S8A and B, ESI $\dagger$ ).

Fig. 7A shows a Raman spectroscopic image of a live NIH/ 3T3 fibroblast with embedded $\mathrm{SiO}_{2}$ @Ag-PAA particles as a colourful spectral map. This image was reconstructed by using the hypercluster analysis (HCA) from the strongest bands that
(A)

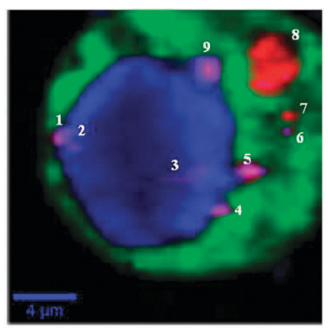

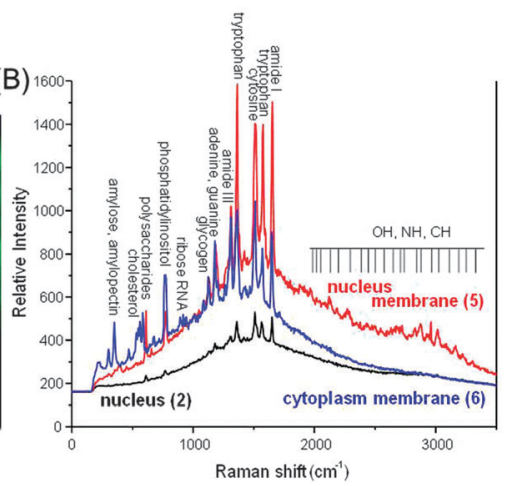

Fig. 7 (A) Raman spectroscopic imaging of a live NIH/3T3 fibroblast with embedded $\mathrm{SiO}_{2} @ \mathrm{Ag}$-PAA particle in a coloured spectral map (scale bar is $4 \mu \mathrm{m})$. The map reflects the differences found in the Raman data and SERS active Ag-PAA nanofilms. This map is a linear combination of the averaged single spectra and characteristic for cell compartments (green cytoplasm and blue - nucleus). Intense green and blue colors of spots separated by a darker background contrast indicate the presence of SERS effective $\mathrm{SiO}_{2}(\mathrm{aAg}-\mathrm{PAA}$ particles inside the cellular medium. Spots with violet and red colors show $\mathrm{SiO}_{2} \mathrm{QAg}-\mathrm{PAA}$ particles that are located at the membrane interface or at its surface (highlighted with numbers). A confocal Raman image is generated by integration of the intensity of the strongest bands in the three spectral ranges: (i) $<1000 \mathrm{~cm}^{-1}$, (ii) $1000-2000 \mathrm{~cm}^{-1}$ and (iii) $2500-3500 \mathrm{~cm}^{-1}$ after local baseline subtraction using the hypercluster analysis (HCA) as one of the efficient label-free methods for the visualization of intracellular components and processes. (B) Selected SERS spectra that are collected from nucleus ( 2 in A) and periphery membranes of the nucleus ( 5 in A) or cytoplasm (6 in A). The laser excitation wavelength was $532 \mathrm{~nm}$ and the grating was $600 \mathrm{~g} \mathrm{~mm}^{-1}$ $(B L Z=500 \mathrm{~nm})$ and spectral resolution of $3 \mathrm{~cm}^{-1}$.

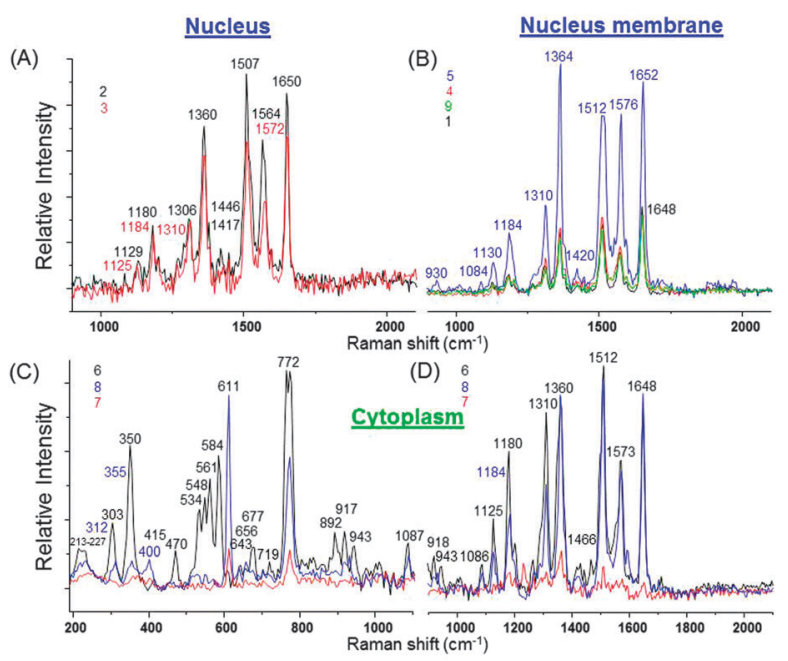

Fig. 8 Cumulated average SERS spectra collected at different locations inside live NIH/3T3 fibroblasts with the numbers indicating position of $\mathrm{SiO}_{2} @ \mathrm{Ag}-\mathrm{PAA}$ particles from the HCA of the SERS image in Fig. 7A. The laser excitation wavelength was $532 \mathrm{~nm}$ and the grating was $600 \mathrm{~g} \mathrm{~mm}^{-1}$ $(B L Z=500 \mathrm{~nm})$ with a spectral resolution of $3 \mathrm{~cm}^{-1}$

are cumulative and averaged from at least ten fibroblasts. To note, the HCA can provide label-free methods for the visualization of intracellular components and processes based on strong Raman/SERS signals (Fig. S9, ESI $\dagger$ ). The confocal laser scanning microscopy image shows the same cell with embedded 
SERS effective $\mathrm{SiO}_{2} @ A g-P A A$ particles that are closely-packed throughout the intracellular interior, being in the nucleus and cytoplasm (Fig. S9A, ESI $\dagger$ ). Colourful regions of the nucleus and cytoplasm are clearly distinguishable by the contrast differences (brighter regions are due to strong SERS activity) (Fig. 7A). The numbered spots with a violet or red color are those closely-packed SERS effective $\mathrm{SiO}_{2} @ A$ A-PAA particles that appear at the membrane interface or at the outer surface of the cell, respectively.

It is well-known that, the cellular nuclei can be distinguished from the cytoplasm, but Raman signals due to DNA are barely visible. This suggests that HCA identifies the nucleus mostly by protein composition (the nuclear proteins differ from cytoplasmic proteins), rather than the actual DNA signal. However, during certain stages of mitosis, the DNA can be so condensed that it exhibits such a strong signal to be relatively easily observable, which is not the case here. In the HCA image analysis the intensity at each pixel is used to threshold the image, which is segmented into pseudocolors. In this way, the intensity is necessary to display those regions of the image corresponding to chemical information and for the computer to correlate or discriminate regions based on spectroscopic data. In our spectral data the intensity is stronger than the necessary threshold value in order to perform the HCA analysis and clearly distinguish the intracellular compartments. To note, the HCA method can be also supported by a reliable technique of principle component analysis (PCA) based on the group discrimination in SERS imaging of organelles. ${ }^{34}$

For analysis we select and number the following locations inside the cell where the SERS intensity is high, i.e. (i) inside the nucleus ( 2 and 3$)$, on its periphery membrane $(1,4,5$, and 9) and inside the cytoplasm (6, 7 and 8) (Fig. 7A). Strong SERS spectra exhibit multiple peaks over the entire spectral region (Fig. 7B) with different shape profiles from the nucleus and periphery membrane of the nucleus or cytoplasm.

Strong SERS signals arise from amide $\left(-\mathrm{CONH}_{2}\right.$, neutral), tryptophan (negatively charged at $6<\mathrm{pH}<8.5$ ), cytosine (negatively charged), adenine (interact with the $\mathrm{Ag}$ surface due to the highly negative charge density of the N1 nitrogen $\left.{ }^{36}\right)$, phosphatidylinositol $\left(\mathrm{COO}^{-}\right)$, polysaccharides $\left(\mathrm{NH}_{3}{ }^{-}, \mathrm{COO}^{-}\right)$, cholesterol (negative, $\mathrm{OH}^{-}, \mathrm{CH}_{3}{ }^{-}$) or amylose (negative, $\mathrm{CH}_{2} \mathrm{OH}, \mathrm{H}$ and $\mathrm{OH}$ ), among others. To note, the SERS spectra of $\mathrm{SiO}_{2} @ \mathrm{Ag}$-PAA particles in culture medium or PBS solution without fibroblasts exhibit small peaks at $788 \mathrm{~cm}^{-1}$ and $965 \mathrm{~cm}^{-1}$ (in CM) or $1321 \mathrm{~cm}^{-1}$ and $1593 \mathrm{~cm}^{-1}$ (in PBS) (Fig. S10, ESI $\dagger$ ) not observable with live fibroblasts (Fig. 7B).

As some fibroblast cell lines prefer slightly more basic environment ( $\mathrm{pH}$ 7.4-7.7), an anionic PAA corona over Ag NPs does not have free carboxylic acid groups, contributing to the electrostatic stabilization of Ag NPs. In this way, aggregation of Ag NPs is unlikely as most citrate ions are not displaced. The $\mathrm{COO}^{-}$groups of the detected molecules can displace original citrate ions of the PAA and come closer to the metallic silver surface. Hence the SERS signals of molecular prints are strong and repeatable, and any analyte effect on the SERS effective $\mathrm{Ag}$-PAA nanofilm is avoided. Moreover, from a SERS point of view the ideal system would be that of a stable solution of small nanoparticle clusters over the microscale surface, perfectly matching stable $\mathrm{SiO}_{2} @ A g-\mathrm{PAA}$ particles. As no aggregates of silver nanoparticles appeared either in the bulk solution of CM or PBS (Fig. S11, ESI $\dagger)$ or inside live fibroblasts $\left(\mathrm{SiO}_{2} @ A g-\mathrm{PAA}\right.$ are only closely packed to each other) undesirable timedependent and irreproducible results can be avoided.

In Fig. 8 multiple strong SERS spectra from intracellular compartments are extensively informative and reproducible. Because of this we decided to group these SERS spectra in three spectral regions in order to perform the intracellular study: (I) $200-1000 \mathrm{~cm}^{-1}$; (II) $1000-2000 \mathrm{~cm}^{-1}$ and (III) $2000-3800 \mathrm{~cm}^{-1}$ (Fig. 8 and Tables S2-S4, ESI $\dagger$ ).

Inside the nucleus the SERS spectra exhibit slight changes in its center or close to the periphery membrane (Fig. 8A). The most prominent SERS peaks appear from tryptophan (strong at $1360 \mathrm{~cm}^{-1}$ and $1564-1572 \mathrm{~cm}^{-1}$ ), cytosine (very strong at $1507 \mathrm{~cm}^{-1}$ ) and amide I (very strong at $1650 \mathrm{~cm}^{-1}$ ), that is important for the dehydration synthesis reaction (condensation reaction) and usually occurs between aminoacids (Table S3, ESI $\dagger$ ). However, at the nucleus membrane these peaks are much stronger (nine fold higher intensity), especially at a hot spot 5, (Fig. 8B) and appear at higher frequency shifts due to the decreased concentration (Table S3, ESI $\dagger$ ). In addition, a strong SERS peak from cytosine, adenine or guanine $\left(1184 \mathrm{~cm}^{-1}\right)$ is observed at seven fold higher intensity, while it appears only as a weak signal inside the nucleus (Fig. 8A and B; Table S3, ESI $†$ ).

It is important to note, that adenine is an interesting molecule in the field of SERS, which interacts with the silver surface at the N1 site due to the highly negative charge density of this site. ${ }^{36}$ It is the first small organic molecule used in single-molecule SERS measurements. ${ }^{35-37}$ This means that SERS effective $\mathrm{SiO}_{2} @ A g-P A A$ particles can be capable to perform single molecule SERS studies of single live cells.

Other important biomolecules such as cholesterol and phosphatidylinositol exhibit stronger SERS prints at the nucleus membrane than inside the nucleus or at its periphery (Table S2, ESI $\dagger$ ). And the extensive $\mathrm{OH}, \mathrm{NH}$ and $\mathrm{CH}$ stretching groups appear as stronger SERS signals from the nucleus membrane rather than from its center (Table S4, ESI†).

It is remarkable, that in the cytoplasm the overall SERS spectra appear with a far larger number of strong signals and from much more biomolecules in all three selected spectral regions (Fig. 8C and D; Tables S2-S4, ESI $\dagger$ ). In particular, very strong SERS signatures from cholesterol (at $534 \mathrm{~cm}^{-1}, 548 \mathrm{~cm}^{-1}$ and $611 \mathrm{~cm}^{-1}$ ), phosphatidylinositol (at $772 \mathrm{~cm}^{-1}$ ) and new moderate signals from polysaccharides, amylose, amylopectin $\left(470 \mathrm{~cm}^{-1}\right)$, ring breathing in the DNA bases $\left(677 \mathrm{~cm}^{-1}\right)$, saccharide band, C-C skeletal backbone $\left(892 \mathrm{~cm}^{-1}\right), \nu(\mathrm{C}-\mathrm{C})$ gauche $\left(1086 \mathrm{~cm}^{-1}\right)$ and antisymmetric phosphate stretching vibration $\left(1230 \mathrm{~cm}^{-1}\right)$ are not observable in the nucleus or at its membrane (Tables S2 and S3, ESI $\dagger$ ). To remind, cholesterol is important for the permeability and fluidity of the membrane. In this way, we may assume that its strong SERS signature arises from the periphery membranes of organelles inside the cytosol. Distinct from the nucleus or its membrane, numerous 
resolved SERS peaks appear from the phospholipids in the region below $800 \mathrm{~cm}^{-1}$ which can be assigned to different residues at the phosphate-ester head-groups. ${ }^{38-40}$

Extensive SERS prints appear with multiple less strong new peaks of symmetric stretching of the choline group, phospholipids $\left(719 \mathrm{~cm}^{-1}\right)^{41}$ ribose vibration, that is one of the distinct RNA modes $\left(918 \mathrm{~cm}^{-1}\right.$ and $975 \mathrm{~cm}^{-1}$ ), $\mathrm{PO}_{2}{ }^{-}$ stretching (DNA/RNA) (1060 $\mathrm{cm}^{-1}$ and $\left.1069 \mathrm{~cm}^{-1}\right), \mathrm{C}=\mathrm{N}$ and $\mathrm{C}=\mathrm{C}$ stretching in a quinoid ring $\left(1594 \mathrm{~cm}^{-1}\right)$ (Tables $\mathrm{S} 2$ and $\mathrm{S} 3$, ESI $\dagger$ ). It is possible to monitor the changes of other components such as glycogen $\left(1047 \mathrm{~cm}^{-1}\right)$, triglycerides in fatty acids with $\delta(=\mathrm{C}-\mathrm{H})$ (phospholipids) (1264-1268 $\left.\mathrm{cm}^{-1}\right)$ and lipids $\left(1466 \mathrm{~cm}^{-1}\right)$ due to the high quality of SERS signals (Tables S2 and S3, ESI $\dagger$ ). Furthermore, multiple arrays of the SERS signatures of $\mathrm{OH}, \mathrm{NH}$ and $\mathrm{CH}$ stretching groups exhibit peaks that can be found either in the nucleus or at its membrane (Table S4, ESI $\dagger$ ).

To remind, the spectral shift indicates changes in the concentration of detected molecules. ${ }^{42,43}$ In our SERS spectra the largest shifts were observed in the $\nu(\mathrm{C}-\mathrm{C})$ stretching in amino acids and skeletal modes of polysaccharides and amylose $\left(930 \mathrm{~cm}^{-1}\right.$ and $\left.943 \mathrm{~cm}^{-1}\right)$, indicating the concentration decrease of the last ones. Less pronounced shifts were examined in the $\nu(\mathrm{C}-\mathrm{C})$ skeletal of the acyl backbone in lipids $\left(1125 \mathrm{~cm}^{-1}\right.$ and $1130 \mathrm{~cm}^{-1}$ ), that correspond to the trans conformation. Fewer molecules can contribute to this vibrational signal inside the nucleus and its corresponding periphery membrane than in the cytosol. A very strong SERS signature from tryptophan $\left(1360 \mathrm{~cm}^{-1}\right)$ appears at higher frequency $\left(1364 \mathrm{~cm}^{-1}\right)$ at the nucleus membrane due to the higher concentration. This is an interesting finding as tryptophan is an amino acid being essential in the human diet, as it was demonstrated by its growth effects on rats. ${ }^{22}$ Overall it is observed that the cytoplasm region shows relatively high concentrations of lipids compared to the nucleus region. Notable nucleic acid related vibrations in the cytoplasm regions are attributed to the ribose contribution, one of the distinct RNA modes.

\section{Conclusions}

In this work the SERS effective nanofilm approach has been advanced by a Ag-PAA matrix which exhibits enhancement factors, EFs $\sim 10^{8}$ more than sufficient for single molecule studies. High EFs can be achieved by the coupling of primary and secondary SERS hot spots of Ag-PAA nanofilm on silica MPs ( $\mathrm{SiO}_{2} @ A g-P A A$ particles). From theoretical calculation in the electrostatic approximation such high local field intensity (LFI) and SERS EFs can arise from prolate Ag-PAA (low aspect ratio) on silica, not in water or with gold (instead of silver). However, at higher aspect ratio the EFs on silver or gold can be comparable. Oblate nanoparticles can exhibit EFs of two orders of magnitude lower than those with prolate shape.

$\mathrm{SiO}_{2} @ A g-P A A$ particles can have a number of superior advantages to achieve a LFI EFs (SERS EFs) increase than a common core-shell model (Ag NPs which is coated by a thin outer silica layer):

- SERS EFs decay very slowly with the distance from the nanofilm surface and still have high values at distances above $10 \mathrm{~nm}$;

- Larger coverage by analytes (up to $10 \mathrm{~nm}$ ) experiencing higher EFs;

- The cumulated SERS intensity saturates slower by at least two fold with the coverage increase;

- The average SERS EFs are higher by two orders of magnitude $\mathrm{Ag}$ oblate nanoparticle, when the incident polarization is along $z$ direction and about ten times larger for $\mathrm{Ag}$ prolate nanoparticles, when the incident polarization is along $x$ direction.

Because of the strong SERS intensity from Ag-PAA nanofilms the hypercluster analysis (HCA) of live single fibroblasts can be carried out in real time. This is important as HCA is one of the most efficient label-free methods for the visualization of intracellular components and processes. SERS bands can be reproducible with multiple strong peaks throughout the whole spectral region with clearly distinguishable shapes from nucleus, periphery membrane and cytosol. As a proof of single molecule detection - adenine can be detected from live fibroblast by a Ag-PAA nanofilm.

Our results demonstrate how live single cells can be chemically imaged with single molecule precision by a SERS effective Ag-PAA nanofilm on silica MPs as reliable substrates. These findings can be of potential practical importance to conduct systematic analyte analysis of a live single cell in real time. Moreover, cell biological aspects such as the intracellular metabolism, drug uptake, apoptosis, and other effects could, in principle, be also investigated.

\section{Experimental part}

\section{Materials}

Silver nitrate $\left(\mathrm{AgNO}_{3}\right.$, analytical grade, $\left.99.8 \%\right)$ was purchased from Serva (Germany). Trisodium citrate dihydrate (99\%) was obtained from Acros Organics Thermo Fischer scientific (Belgium). Polyacrylic acid sodium salt (PAA, $M_{\mathrm{W}} \approx 5100 \mathrm{Da}$ ), polyvinyl pyrrolidone (PVP K $\left.30, M_{\mathrm{W}} \approx 55000 \mathrm{Da}\right)$, rhodamine 6G (99\%) and 3-aminopropyltriethoxysilane (APTES) were purchased from Aldrich. Silica particles $\left(\mathrm{SiO}_{2}, 1.55 \pm 0.04 \mu \mathrm{m}, 50 \mathrm{mg} \mathrm{mL}{ }^{-1}\right)$ were obtained from Microparticles $\mathrm{GmbH}$ (Germany). Methanol was purchased from Merck KGaA (Germany) and toluene was obtained from AppliChem (Germany). Sodium chloride ( $\mathrm{NaCl}, \geq 99.5 \%$, biograd, suitable for the cell culture medium), DMEM, gentamicin, glucose and phosphate buffered saline (PBS, bioperformance certified, suitable for cell culture) were purchased from Sigma-Aldrich Co. (Germany). Calf serum was purchased from PAA Laboratories $\mathrm{GmbH}$ (Austria).

The water used in all the experiments was prepared in a three-stage Millipore Milli-Q Plus 185 purification system and had a resistivity higher than $18.2 \mathrm{M} \Omega \mathrm{cm}^{-1}$. For the experiments with living cells Milli-Q water was autoclaved.

\section{Preparation of silver nanoparticles (Ag NPs)}

(a) Formation by Meisel's method ${ }^{23}$. Aqueous solution of silver nitrate $\left(1 \times 10^{-3} \mathrm{~mol} \mathrm{~L}^{-1}\right)$ was bubbled with nitrogen gas 
in a $0.5 \mathrm{~L}$ flask and heated up to the boiling point under vigorous stirring $(650 \mathrm{rpm}) \cdot 10^{-2} \mathrm{~L}$ of $1 \%$ of trisodium citrate dihydrate aqueous solution was purged with nitrogen gas and dropwise added under vigorous stirring (450 rpm). After one hour the heat source was removed and the reaction mixture was stirred for another $30 \mathrm{~min}$. The final silver colloidal solution appeared milky white-grey and the concentration of silver nanoparticles was $0.73 \times 10^{-3} \mathrm{~mol} \mathrm{~L}^{-1}$ at $\mathrm{pH}=8.5$. The nanoparticle's surface charge was $-22 \mathrm{mV}$ (average standard deviation $5 \mathrm{mV}$ ). The final silver colloidal solution was kept in darkness at $4{ }^{\circ} \mathrm{C}$ and no sediment was observed within months.

(b) Formation by Xia's method ${ }^{26}$. Before reaction $47.5 \times$ $10^{-3}$ L Milli-Q water was bubbled with nitrogen gas under vigorous stirring with a magnetic bar (600 rpm). After $30 \mathrm{~min}$ $0.5 \times 10^{-3} \mathrm{~L}$ of aqueous solution of trisodium citrate dihydrate $\left(30 \times 10^{-3} \mathrm{~mol} \mathrm{~L}^{-1}\right)$ and $1 \times 10^{-3} \mathrm{~mol} \mathrm{~L}^{-1}$ of silver nitrate $\left(5 \times 10^{-3} \mathrm{~mol} \mathrm{~L}^{-1}\right)$ were quickly added under vigorous stirring (1200 rpm). When $0.5 \times 10^{-3} \mathrm{~L}$ of freshly prepared aqueous solution of sodium borohydride $\left(50 \times 10^{-3} \mathrm{~mol} \mathrm{~L}^{-1}\right)$ was added, the reaction mixture turned into a light yellow color and was left with stirring for the next $30 \mathrm{~min}$. When $0.5 \times 10^{-3} \mathrm{~L}$ of aqueous solution of polyvinyl pyrrolidone $\left(5 \mathrm{mg} \mathrm{mL} \mathrm{mL}^{-1}\right)$ was added, the reaction solution became dark yellow. After the next $30 \mathrm{~min} 20 \times 10^{-3} \mathrm{~L}$ of the reaction mixture was transferred into a $50 \mathrm{~mL}$ flask placed in a thermo bath at $80{ }^{\circ} \mathrm{C}$ for 3 hours. The final silver colloidal solution was intense yellow-green and was kept in darkness at $4{ }^{\circ} \mathrm{C}$. No sediment and no color change of silver nanoparticles were observed within a month. The nanoparticle's surface potential was $-27 \mathrm{mV}$ (the average standard deviation 8). The concentration of silver sols was estimated from the UV-Vis absorbance spectra by the Beer-LambertBouguer law as $0.58 \times 10^{-3} \mathrm{~mol} \mathrm{~L}^{-1}(\mathrm{pH}=8.4)$.

(c) Preparation of aqueous solution of polyacrylic acid. Aqueous solution of polyacrylic acid (PAA) was prepared with a typical concentration $2 \mathrm{mg} \mathrm{mL} \mathrm{mL}^{-1}$ in $0.5 \mathrm{M} \mathrm{NaCl}$ followed by purification in a sealed semipermeable membrane (cellulose acetate) against MQ water $\left(M_{\mathrm{W}}\right.$ cut off $\left.20 \mathrm{kDa}\right)$ and lyophilized. PAA $(\mathrm{pH}=7.6)$ aqueous solution was obtained under vigorous stirring at room temperature.

(d) Formation of Ag-PAA nanofilm. Aqueous solution of $\mathrm{Ag}$ NPs was added to the volume excess of PAA aqueous solution at a ratio $1: 4$ at room temperature in darkness. The Ag-PAA colloidal mixture was incubated at mild shaking $(300 \mathrm{rpm})$ in an Eppendorf Thermomixer compact (Hamburg, Germany) at $37^{\circ} \mathrm{C}$ for $20 \mathrm{~min}$, then triply washed by centrifugation $(14000 \mathrm{rpm}$ for $30 \mathrm{~min}$ ). The Ag-PAA surface potential was $-45 \pm 5 \mathrm{mV}$.

(e) Preparation of positively charged silica microparticles. Before treatment the colloidal silica suspension $\left(5 \mathrm{mg} \mathrm{mL}^{-1}\right)$ was triply washed with Milli-Q water by repetitive centrifugation at $8000 \mathrm{rpm}$. The initially negative surface potential of silica $(-22.4 \pm$ $5 \mathrm{mV}$ in pure water) was reversed by functionalizing the hydroxyl groups with aminosilanes. ${ }^{22,23} 100 \mathrm{mg}$ of silica particles were transferred from water to toluene by washing them twice with methanol followed by toluene by centrifugation at $8000 \mathrm{rpm}$ for $5 \mathrm{~min}$. The particles were then dispersed in $10 \mathrm{~mL}$ of toluene by stirring, and $1 \mathrm{~mL}$ 3-aminopropyltriethoxysilane (APTES) was added dropwise under nitrogen atmosphere. The mixture was stirred for 1 hour at room temperature. Positively charged silica particles were washed three times with toluene, methanol, and water consecutively in order to remove excess APTES and to transfer particles back into aqueous bulk. The washing of particles was carried out by centrifugation cycles at room temperature and $8000 \mathrm{rpm}$ for $20 \mathrm{~min}$. The surface potential of the silica particles was $+44 \pm 5 \mathrm{mV}$.

(f) Deposition of Ag-PAA nanofilm on silica particles. Positively charged silica particles were added to aqueous solution of Ag NPs at a volume ratio 1:9 and incubated at room temperature for $15 \mathrm{~min}$ at mild shaking $300 \mathrm{rpm}$. The silica-silver mixture was triply washed with Milli Q autoclaved water by centrifugation ( $8000 \mathrm{rpm}$ for $5 \mathrm{~min}$ ). The adsorption procedure was repeated three times. The average surface potential of the $\mathrm{SiO}_{2} @ A g-\mathrm{PAA}$ particles was $-45 \pm 5 \mathrm{mV}$.

(g) Measurements of the SERS efficiency of Ag-PAA solutions. The stock Rh6G solution was prepared at a concentration of $10^{-3} \mathrm{~mol} \mathrm{~L}^{-1}$ in MQ water. Lower concentration of $10^{-6} \mathrm{~mol} \mathrm{~L}^{-1}$ was obtained by successive dilution of the stock solution by a factor of 10 and 100 . The dye concentration was monitored via the fluorescence intensity with a calibration curve (intensity linearly decreased with the decreased concentration of dye molecules). For Raman measurements fresh aqueous solution of $\mathrm{SiO}_{2} @ A g-\mathrm{PAA}$ particles was added to a $10^{-4} \mathrm{~mol} \mathrm{~L}^{-1} \mathrm{NaCl}$ solution at a volume ratio 1:5 of salt to sol followed by the addition of fresh $1 \mu \mathrm{L}$ Rh6G solution. The fluorescence of $\mathrm{SiO}_{2} @ A g-\mathrm{PAA}$ particles with adsorbed dye molecules was quenched due to electron transfer (Fig. S4, ESI $\dagger$ ).

(h) Cell culture and electroporation of fibroblasts. Fibroblasts NIH/3T3 (purchased from DMSZ) were cultured in Dulbeco's modified eagle's medium (DMEM) supplemented with $4.5 \mathrm{~g} \mathrm{~L}^{-1}$ glucose, $10 \mathrm{v} \%$ calf serum, $10^{-2} \mathrm{~g} \mathrm{~L}^{-1}$ gentamicin (antibiotic). Cells were seeded with $3 \times 10^{3}$ cells $\mathrm{cm}^{-2}$ on the glass bottom dish (purchased from Greiner Bio One) $8.8 \mathrm{~cm}^{2}$ culture surface and incubated (Binder) with $5 \% \mathrm{CO}_{2}$ at $37{ }^{\circ} \mathrm{C}$ overnight before Raman measurements.

$\mathrm{SiO}_{2} @ A g-\mathrm{PAA}$ particles were delivered into the cellular interior by the electro-permeabilization of the cell membranes in a BIORAD gene-pulser cuvette with $0.4 \mathrm{~cm}$ electrode gap inserted inside a home-made electric cell connected to the GHT-Bi500 electroporator ( $\beta$ tech, France). $200 \times 10^{-6} \mathrm{~L}\left(5.34 \times 10^{4}\right.$ cells $)$ in a culture medium were mixed with $400 \times 10^{-6} \mathrm{~L}$ of silver colloidal solution at a sequence of pulse voltages of $250 \mathrm{~V}$ for the positive and negative parts with a pulse length $500 \mathrm{~ms}$ within the sequence. After electroporation the colloidal mixture with the cell suspension was mixed with $1 \times 10^{-3} \mathrm{~L}$ of culture medium and deposited on to the glass bottom of a petri dish in the incubator. The inspection of the incubated cells was checked by optical microscopy. Before Raman measurements the cells were washed at least three times with PBS aqueous solution.

\section{Characterization}

(a) UV-Vis absorption, zeta potential, dynamic light scattering, electron/optical microscopy and fluorescence. For size characterization of particles, a Zeiss EM 912 Omega transmission electron 
microscope (TEM) and a high-performance particle sizer (Malvern Instruments) for dynamic light-scattering (DLS) measurements were employed. Light absorption of particles was collected by a Varian CARY50 Conc UV-Vis spectrophotometer in the wavelength range from 200 to $900 \mathrm{~nm}$ in a quartz cell with $10 \mathrm{~mm}$ path length. The $\zeta$-potential of particles was measured using a zeta sizer (Malvern Instruments). The fluorescence measurements were performed by a FluoroMax-4 spectrofluorometer (Horiba Jobin Yvon) in the UV-Vis spectral range. A Nikon Eclipse TS100 routine inverted microscope was employed to collect the refracted light from the transparent live cells through the enhancement of their phase contrast. Observation of the cell's morphology was conducted by a Leica TCS SP inverted confocal laser scanning microscope system (CLSM Leica, Germany) equipped with a $40 \times$ oil immersion objective having 1.25 numerical aperture and $0.1 \mathrm{~mm}$ working distance.

(b) Raman spectroscopy and microscopy. Raman and surface enhanced Raman scattering (SERS) spectra were collected by two confocal Raman microscopes. The first one was a confocal Raman microscope (CRM200, WITec, Ulm, Germany) equipped with a piezo-scanner (P-500, Physik Instrumente, Karlsruhe, Germany) working at a $785 \mathrm{~nm}$ excitation wavelength (Toptica Photonics AG, Graefelfing, Germany). A linearly polarized diode laser beam was focused through the LWD $20 \times$, Nikon Fluor $60 \times$ water immersion and Nikon $100 \times$ oil immersion objectives with numerical apertures $N_{\mathrm{A}}=0.40,1.00$ and 1.25 , respectively. The laser power was kept no higher than $10 \mathrm{~mW}$ as measured by a Newport optical power meter $1830 \mathrm{C}$. The scattered light was filtered with an analyzer (further polarizer) through the confocal microscope pinhole. The spectra were collected with a $300 \mathrm{~g} \mathrm{~mm} \mathrm{~mm}^{-1}$ grating blazed at $750 \mathrm{~nm}$ and recorded by a spectrograph (Acton, Princeton Instruments Inc., Trenton, NJ, USA) with a cooled CCD detector (PI-MAX, Princeton Instruments Inc., Trenton, NJ, USA), with an integration time $1 \mathrm{~s}$ of fifty accumulations and spectral resolution of $6 \mathrm{~cm}^{-1}$.

The second confocal Raman microscope (alpha 300, WITec, Ulm, Germany) was employed with a frequency doubled Nd:YAG (532 nm) laser excitation and piezoscanner (P-500, Physik Instrumente, Karlsruhe, Germany). The spectra were acquired with a thermoelectrically cooled CCD detector (DU401A-BV, Andor, UK), with an integration time $1 \mathrm{~s}$ of fifty accumulations with a grating $600 \mathrm{~g} \mathrm{~mm}^{-1}(\mathrm{BLZ}=500 \mathrm{~nm})$ and spectral resolution of $3 \mathrm{~cm}^{-1}$. The excitation laser intensity was in the range from $7.9 \mathrm{~kW} \mathrm{~cm}^{-2}$ to $23.8 \mathrm{~kW} \mathrm{~cm} \mathrm{~cm}^{-2}$ (i.e. laser power from $1 \mathrm{~mW}$ to $3 \mathrm{~mW}$ ) with a spectral resolution of $3 \mathrm{~cm}^{-1}$.

The signal to noise ratio was high enough to ensure the low value of the root mean square fluctuations averaged in time (typically below 5\%). Basic alignment was carried out by a Raman spectrum of a silicon wafer with a characteristic Si line at $520 \mathrm{~cm}^{-1}$ during integration times from 0.3 to $1 \mathrm{~s}$. The SERS measurements of live cells were conducted in real time in a $\mu$-dish (35 mm, Ibidi, Munich, Germany) equipped with a heating stage and external temperature probe (Bioscience Tools, USA) to maintain $37^{\circ} \mathrm{C}$ of the cells. The acquired Raman and SERS spectra were corrected for the baseline, background of the $\mu$-dish substrate and the $\mathrm{SiO}_{2}\left(485 \mathrm{~cm}^{-1}\right.$ band). For imaging integration time of $0.1 \mathrm{~s}$ per pixel was used. The Scan
Ctrl Spectroscopy Plus software (version 1.38, WITec) was used for measurement, WITec Project Plus (version 2.02, WITec) for spectra processing. Raman images were generated with the WITec software using a sum filter, which integrates the intensity of the signal for a defined wavenumber range of interest and subtracts the background as a linear baseline from the upper to lower border. Hypercluster analysis (HCA) of Raman images was performed with the OPUS 6.5 software. Briefly, HCA was used to sort objectives according to a defined similarity based on Raman/SERS spectra. HCA builds the cluster by combining the objectives, which are treated and linked into larger groups by calculating criterion (spectral bands and regions) distance step by step.

\section{(c) Experimental estimation of SERS EFs.}

$$
\mathrm{EF}=\frac{I_{\text {SERS }}}{I_{\text {norm }}} \cdot \frac{C_{\text {norm }}}{C_{\text {SERS }}}
$$

where EF is the average enhancement factor (averaged over all possible positions on the metallic surface and also from molecules randomly adsorbed on the surface as compared to the same number of non-adsorbed molecules), $C_{\text {norm }}$ and $C_{\text {SERS }}$ are concentrations of Rh6G aqueous solutions for SERS and normal Raman measurement (i.e. number of molecules on the surface of a single nanoparticle and in bulk solution effectively excited by the laser beam); $I_{\text {SERS }}$ and $I_{\text {norm }}$ are corresponding intensities of these vibrational modes. The Raman peak of Rh6G at $1365 \mathrm{~cm}^{-1}$ was taken for the EF estimation. To estimate the molecular density of Rh6G adsorbed on Ag NP (average radius $15 \times 10^{-9} \mathrm{~m}$ ) surface we consider the aqueous solution of $\mathrm{Ag} \mathrm{NPs}$ at $0.58 \times$ $10^{-3} \mathrm{~mL}^{-1}$ concentration in a volume $0.6 \mathrm{~mL}$ incubated with $10^{-8} \mathrm{~mol} \mathrm{~L}^{-1}$ Rh6G aqueous solution. The mole number of silver is $\nu_{\mathrm{Ag}}=0.3 \times 10^{-6} \mathrm{~mol}$

$$
n_{\mathrm{Ag}}=\frac{N_{\mathrm{Ag}}}{V}, N_{\mathrm{Ag}}=\nu_{\mathrm{Ag}} \cdot N_{\mathrm{A}},
$$

where $n_{\mathrm{Ag}}$ is the molecular density of silver, $N_{\mathrm{Ag}}$ is the number of silver atoms in a volume, $N_{\mathrm{A}}$ is Avogadro's number $(6.02 \times$ $\left.10^{23} \mathrm{~mol}^{-1}\right)$. The total average density of silver is $3.48 \times$ $10^{20} \mathrm{~L}^{-1}$ and of Rh6G molecules is $6.02 \times 10^{15} \mathrm{~L}^{-1}$. As the silver crystals with the atoms are arranged in the face-centred cubic stacking cell with about four atoms, $N$, inside the unit cell for each of the packing arrangements, the $74 \%$ packing efficiency, $h$, as follows

$$
h=N \cdot \frac{V_{\text {sphere }}}{V_{\text {total }}}=\frac{4 \cdot 4 / 3 \pi r^{3}}{16 \cdot \sqrt{2} r^{3}},
$$

where $V_{\text {sphere }}$ and $V_{\text {total }}$ are the volume occupied by a sphere and a total volume, $r$ is a radius of an atom. The covalent radius of a silver atom is $145 \pm 5 \times 10^{-12} \mathrm{~m}$ and its volume is $1.28 \times 10^{-29} \mathrm{~m}^{3}$ (spherical geometry). The radius of a particle is $15 \times 10^{-9} \mathrm{~m}$ and the volume of a spherical particle is $1.41 \times$ $10^{-23} \mathrm{~m}$. In this way, taking the packing efficiency into account,

$$
N_{\text {atoms }}=N \cdot \frac{V_{\text {particle }}}{V_{\text {atom }}},
$$


one single Ag NP consists of $8.15 \times 10^{5}$ silver atoms. The average silver density is $4.3 \times 10^{14} \mathrm{~L}^{-1}$ and when it is divided by the dye density, we have about 14 Rh6G molecules adsorbed on one single Ag NP.

(d) Theoretical calculation of SERS EF versus distance and coverage. All calculations have been carried out in MATLAB R2012 software and basic code scripts were taken from elsewhere. ${ }^{44}$ For calculation we adapted the theory to multi-layers of spheres with concentric interfaces and applied the simplest example, which is a coated sphere. We consider $V_{0}$ as the volume of a sphere, and $V_{\mathrm{K}}$ as the outside (embedding) medium for the single sphere. Each volume is described by a dielectric function $\varepsilon_{M}$ with the wave vector in each region defined as

$$
k=\sqrt{\varepsilon_{\mathrm{M}}} \omega / c,
$$

As for a single sphere, the electric field solution is expanded for each spherical symmetry in every volume. The extension of Mie theory (generalized Mie theory, GMT) is applied considering two spheres and each of them contributes to a scattering wave that is seen by the other one. The electromagnetic response of the spheres interferes with each other through the respective scattered fields. The scattered field has a contribution from spherical waves (VSHs) emanating from two individual spheres. For a system of two spheres the scattered field is expanded as

$$
E_{\mathrm{sca}}(r)=E_{0} \sum_{i=1}^{N} \sum_{n, m} C_{n, m}^{i} M_{n m}^{(3)}\left(k_{\mathrm{M}}, r-r_{i}\right)+d_{n m}^{i} N_{n m}^{(3)}\left(k_{\mathrm{M}}, r-r_{i}\right),
$$

where $i=1,2$.

The scattered fields are a superposition of VSHs originating from the centers of two different spheres with the interaction of the respective VSHs aligned along $z$ due to the symmetry. For a system of two spheres the internal field is expanded as

$$
E_{\text {int }}(r)=E_{0} \sum_{n=0}^{\infty} \sum_{m=-n}^{m=n} a_{n m} M_{n m}^{(i)}(k, r)+b_{n m} N_{n m}^{(i)}(k, r),
$$

$E_{0}$ - complex electric field amplitude, $a_{n m}$ and $b_{n m}$ non-dimensional complex coefficients, $n$ - positive integer (physical meaning is the total angular momentum along $z$ axis) and $|m| \leq n$ is a projection of this angular momentum along the same axis.

This interpretation is a direct consequence of the spherical symmetry and is completely analogous to the same classification done in quantum mechanics for potentials with spherical symmetry. Vectors $M_{n m}^{(i)}(k, r)$ and $N_{n m}^{(i)}(k, r)$ are called vector spherical harmonics (VSHs) or multipole fields and have the following physical meaning: magnetic and electric field that is created by a given multipolar distribution of sources at the origin. $M_{n m}^{(i)}(k, r)$ describes a magnetic field of an electric dipole and $N_{n m}^{(i)}(k, r)$ determines an electric field of that dipole. For $n>1$ they correspond to the electric fields of higher order electric and magnetic multipoles (quadrupole, etc.). The angular dependence of the VSHs is described in terms of spherical harmonics, while their $r$-dependence involves spherical Bessel functions.

$$
M_{n m}(k, r)=\Delta \times\left(\varsigma_{n m}(k, r), r\right)
$$

and

$$
\begin{gathered}
N_{n m}(k, r)=\frac{1}{k} \Delta \times M_{n m}(k, r) \\
\varsigma_{n m}(k, r)=\frac{1}{\sqrt{n(n+1)}} z_{n}(k r) Y_{n m}(\theta, \phi),
\end{gathered}
$$

where $Z_{n}$ - spherical Bessel functions, $Y_{n m}(\theta, \varphi)$ - spherical harmonics with the following standard convention:

$$
Y_{n m}(\theta, \phi)=\sqrt{\frac{2 n+1(n-m) !}{4 \pi(n+m) !}} P_{n}^{m}(\cos (\theta)) \mathrm{e}^{i m \phi},
$$

where $P_{n m}(\cos (\theta))$ are the associated Legendre functions, defined using the Condon-Shortley phase.

$$
P_{n}^{m}(x)=\frac{(-1)^{m}}{2^{n} n !}\left(1-x^{2}\right)^{\frac{m}{2}} \frac{d^{n+m}}{d x^{n+m}}\left(x^{2}-1\right)^{n}, \quad m \geq 0 .
$$

Unknown complex coefficients must be determined by imposing two independent EM boundary conditions at the sphere surface $(r=a)$. For the scattered field

$C_{n m}=\Gamma_{n} a_{n m}$ and $d_{n m}=\Delta_{n} b_{n m}$, while for the internal field the coefficients are $\alpha_{n m}=A_{n} a_{n m}$ and $\beta_{n m}=B_{n} b_{n m}$.

All of these coefficients are frequency-dependent and their frequency-dependence describes all aspects of the wavelengthdependent optical response of the sphere (i.e. optical resonances).

For small spheres

$$
\Delta_{1}=\frac{2 i}{3} x^{3} \frac{s^{2}-1}{s^{2}+2}\left[1-\frac{3}{5} x^{2} \frac{s^{2}-2}{s^{2}+2}-\frac{2}{3} i x^{3} \frac{s^{2}-1}{s^{2}+2}\right]^{-1}
$$

(up to order $x^{6}$ ) and

$$
\Gamma_{1}=\frac{i}{45} x^{5}\left(s^{2}-1\right)\left[1-\frac{1}{21} x^{2}\left(2 s^{2}-5\right)\right]^{-1}
$$

with

$$
\Delta_{2}=-\frac{i}{30} x^{5} \frac{s^{2}-1}{s^{2}+\frac{3}{2}}\left[1+\frac{5}{14} x^{2} \frac{1}{s^{2}+\frac{3}{2}}\right]^{-1}
$$

We use analytical formulas of the electrostatic approximation (ESA) for a qualitative understanding of scattering field enhancements at the silica/silver interface. We assume that the electric field outside the sphere is created by an electrostatic dipole at the sphere center.

(e) Theoretical calculation of the local field intensity (LFI) EFs. Here we apply the analytical solution for a metallic ellipsoid ( $a \geq b \geq c>0$ along its main axis $x$ or $z$ ) in the ESA. ${ }^{44}$ We consider the LFI EF at the corners on the ellipsoid surface along two main axes. The oblate $(3,1)$ or prolate $(1,3)$ spheroids with the aspect ratio 3 (i.e. $h=a / c$ ) are examined. 
We calculate the LFI EF and SERS EF normal (perpendicular) and tangential (parallel) to the spheroids at any point $r$ on their surface.

$(\text { LFI EF })^{\mathrm{obl}}=M_{\mathrm{loc}}^{\mathrm{obl}}=\left\langle\left|e_{\xi} \cdot e_{z}\right|^{2}\right\rangle=\frac{1}{e_{0}^{2}} \cdot \frac{1-f_{0}}{1+f_{0}}$ with the surface average $f_{0}=\frac{1-e_{0}^{2}}{2 e_{0}} \ln \left(\frac{1+e_{0}}{1-e_{0}}\right)$ and surface area $S=$ $2 \pi a^{2}\left[1+f_{0}\right]$ and $(\mathrm{SERS} \mathrm{EF})^{\mathrm{obl}}=F_{\mathrm{E} 4}^{\mathrm{obl}}=\left(M_{\mathrm{loc}}\right)^{2}=\left\langle\left|e_{\xi} \cdot e_{z}\right|^{4}\right\rangle=$ $\frac{1}{e_{0}{ }^{4}} \cdot \frac{3-2 e_{0}^{2}-3 f_{0}}{1+f_{0}}$ for oblate spheroid $(\mathrm{LFIEF})^{\mathrm{prol}}=M_{\mathrm{loc}}^{\mathrm{prol}}=$ $\left\langle\left|e_{\xi} \cdot e_{z}\right|^{2}\right\rangle=\frac{1-e_{\mathrm{p}}^{2}}{e_{\mathrm{p}}^{2}} \cdot \frac{f_{\mathrm{p}}-1}{1+f_{\mathrm{p}}}$ with the surface average $f_{\mathrm{p}}=$ $\frac{1}{e_{\mathrm{p}} \sqrt{1-e_{\mathrm{p}}^{2}}} \arcsin \left(e_{\mathrm{p}}\right)$ and surface area $S=2 \pi b^{2}\left[1+f_{\mathrm{p}}\right]$ and $(\mathrm{SERS} \mathrm{EF})^{\mathrm{prol}}=F_{\mathrm{E} 4}^{\mathrm{prol}}=\left(M_{\mathrm{loc}}\right)^{2}=\left\langle\left|e_{\xi} \cdot e_{z}\right|^{4}\right\rangle=\frac{3}{8 e_{\mathrm{p}}^{4}}$. $\frac{3-2 e_{\mathrm{p}}^{2}-\left(3-4 e^{2}\right) f_{\mathrm{p}}}{1+f_{\mathrm{p}}}$ for prolate spheroid.

We assume for axes with "flat" curvature $\left(L_{i}>1 / 3\right)$ the resonant wavelength is blue shifted compared to the sphere, but remains bound by the bulk plasmon resonance. For axes with "pointy" curvature $\left(L_{i}<1 / 3\right)$ the resonant wavelength is red shifted compared to the sphere and the amount of the redshift depends on the magnitude of the coefficient is $1 / L_{i}-1$, i.e. the smaller the $L$ value (more pronounced corners of the prolate spheroid) the larger the red-shift.

$$
M_{\mathrm{loc}}^{\mathrm{perp}}(r, \omega)=A^{\mathrm{perp}}(\omega)\left|e_{\xi} \cdot e_{z}\right|^{2},
$$

where

$$
\begin{gathered}
A^{\text {perp }}(\omega)=|1+(3-3 L) \beta(\omega)|^{2}, \\
M_{\mathrm{loc}}^{\text {para }}(r, \omega)=A^{\text {para }}(\omega)\left[1-\left|e_{\xi} \cdot e_{z}\right|^{2}\right],
\end{gathered}
$$

where

$$
A^{\mathrm{para}}(\omega)=|1-3 L \beta(w)|^{2},
$$

where $\beta(\omega)$ is a non-dimensional parameter of polarizability of the metal/dielectric interface, which is defined as

$$
\beta=\frac{\varepsilon(\omega)-\varepsilon_{\mathrm{M}}}{\varepsilon(\omega)+2 \varepsilon_{\mathrm{M}}}
$$

where $\varepsilon$ and is $\varepsilon_{M}$ are dielectric constants in the metal and the medium.

The geometrical or depolarization factors $L_{i}(i=1,3$ correspond to $x$ and $z$ ) for oblate spheroids are $L_{1}=L_{2}<1 / 3<L_{3}$ and prolate spheroids are $L_{1}<1 / 3<L_{2}=L_{3}$. They are defined as following for an oblate spheroid:

$$
L_{1}=L_{2}=\frac{1}{2 e_{0}^{2}}\left[\frac{\sqrt{1-e_{0}^{2}}}{e_{0}} \arcsin \left(e_{0}\right)-\left(1-e_{0}^{2}\right)\right]
$$

and

$$
L_{3}=1-2 L_{1}=\frac{1}{2 e_{0}^{2}}\left[1-\frac{\sqrt{1-e_{0}^{2}}}{e_{0}} \arcsin \left(e_{0}\right)\right]
$$

and prolate spheroid:

$$
L_{1}=\frac{1-e_{\mathrm{p}}^{2}}{e_{\mathrm{p}}^{2}}\left[-1+\frac{1}{2 e_{\mathrm{p}}} \ln \left(\frac{1+e_{\mathrm{p}}}{1-e_{\mathrm{p}}}\right)\right]
$$

and $L_{3}=L_{2}=\frac{1-L_{1}}{2}=\frac{1}{2 e_{\mathrm{p}}^{2}}\left[1-\frac{1-e_{\mathrm{p}}^{2}}{2 e_{\mathrm{p}}} \ln \left(\frac{1+e_{\mathrm{p}}}{1-e_{\mathrm{p}}}\right)\right]$.

$$
M_{\mathrm{loc}}(r, \omega)=A^{\mathrm{para}}(\omega)+\left(A^{\mathrm{perp}}(\omega)-A^{\mathrm{para}}(\omega)\right)\left|e_{\xi}-e_{z}\right|^{2}
$$

with $\left|e_{\xi} \cdot e_{z}\right|^{2}=\frac{z^{2}}{c^{4}\left(x^{2} / a^{4}+y^{2} / b^{4}+z^{2} / c^{4}\right)}$.

$A^{\text {perp }}(\omega)$ and $A^{\text {para }}(\omega)$ are maximum and minimum LFI EF for incident polarization along the electric field $e_{i}$. The maximum enhancement is obtained at two points on this axis (the tips) and the local field is normal to the surface at these points. The minimum enhancement is obtained at four points on two other axes (perpendicular to the incident polarization) and is then tangential to the surface. For other points the wavelength dependence of the LFI EF is determined by $A^{\text {perp }}(\omega)$ and $A^{\text {para }}(\omega)$, while the position dependence is governed by the factor $\left|e_{\xi} \cdot e_{i}\right|^{2}$, where $i=x$ or $z$. For SERS red-shifted resonances are the most important and they can be excited with polarization along the most "pointy" axes.

\section{Acknowledgements}

We thank Rona Pitschke and Heike Runge (Departments of Colloids at MPI) for electron microscopy analysis. We appreciate the allowance to use equipment of the high voltage electroporator from Dr Ronald Knorr and Prof. Rumiana Dimova (Theory Department at MPI). The help with the cell culture assistance by Christine Pilz (Department of Biomaterials at MPI) is greatly appreciated. Research grants from the MaxPlanck society and Alexander von Humboldt foundation are greatly acknowledged.

\section{Notes and references}

1 D. Willis, K. W. Li, J. Q. Zheng, J. H. Chang, A. Smit, T. Kelly, T. T. Merianda, J. Sylvester, J. van Minnen and J. L. Twiss, J. Neurosci., 2005, 25, 778.

2 S. L. Spencer, S. Gaudet, J. G. Albeck, J. M. Burke and P. K. Sorger, Nature, 2009, 459, 428.

3 N. Lafuente, V. Azcutia, N. Matesanz, E. Cercas, L. Rodriguez-Manas, C. F. Sanchez-Ferrer and C. Peiro, J. Cardiovasc. Pharmacol., 2005, 45, 193.

4 M. Mir, A. Bergamaschi, B. S. Katzenellenbogen and G. Popescu, PLoS One, 2014, 9, 1.

5 W. K. Huh, J. V. Falvo and L. C. Gerke, Nature, 2003, 425, 686.

6 A. Jean-Jacques and S. Trajkovska, Fluoresc. Spectrosc., 2006, 7, 1067.

7 M. Hedegaard, C. Matthaus and S. Hassing, Theor. Chem. Acc., 2011, 130, 1249.

8 J. Ando, K. Fujita, N. I. Smith and S. Kawata, Nano Lett., 2011, 11, 5344 . 
9 M. Fleischmann, P. J. Hendra and A. J. Mcquillan, Chem. Phys. Lett., 1974, 26, 163.

10 M. G. Albrecht and J. A. Creighton, J. Am. Chem. Soc., 1977, 99, 5215.

11 D. L. Jeanmaire and R. P. Van Duyne, J. Electroanal. Chem., 1977, 84, 1.

12 S. Emory and S. M. Nie, Anal. Chem., 1997, 69, 2631.

13 J. P. Camden, J. A. Dieringer, Y. Wang, D. J. Masiello, L. D. Marks, G. C. Schatz and R. P. Van Duyne, J. Am. Chem. Soc., 2008, 130, 12616.

14 E. A. Vitol, Z. Orynbayeva, M. J. Bouchard, J. AzizkhanClifford, G. Friedman and Y. Gogotsi, ACS Nano, 2009, 3, 3529.

15 J. Feng Li, X. D. Tian, S. B. Li, J. R. Anema, Z. L. Yang, Y. Ding, Y. F. Wu, Y. M. Zeng, Q. Z. Chen, B. Ren, Z. L. Wang and Z. Q. Tian, Nat. Protoc., 2013, 8, 52.

16 F. Tam, A. L. Chen, J. Kundu, H. Wang and N. J. Halas, J. Chem. Phys., 2007, 127, 204703.

17 K. N. Yu, S. M. Lee, J. Y. Han, H. Park, M. A. Woo, M. S. Noh, S. K. Hwang, J. T. Kwon, H. Jin, Y. K. Kim, P. J. Hergenrother, D. H. Jeong, Y. S. Lee and M. H. Cho, Bioconjugate Chem., 2007, 18, 1155.

18 D. Radziuk and H. Moehwald, Nanoscale, 2014, 6, 6115.

19 A. F. McCabe, C. Eliasson, R. A. Prasath, A. HernandezSantana, L. Stevenson, I. Apple, P. A. G. Cormack, D. Graham, W. E. Smith, P. Corish, S. J. Lipscomb, E. R. Holland and P. D. Prince, Faraday Discuss., 2006, 132, 303.

20 A. Yashchenok, A. Masic, D. Gorin, B. S. Shim, N. A. Kotov, P. Fratzl, H. Moehwald and A. Skirtach, Small, 2013, 9, 351.

21 B. M. DeVetter, S. T. Sivapalan, D. D. Patel, M. V. Schulmerich, C. J. Murphy and R. Bhargava, Langmuir, 2014, 30, 8931-8937.

22 L. Novotny and B. Hecht, Principles of Nano-optics, Cambridge University Press, 2006, ch. 12, pp. 378-418.

23 P. C. Lee and D. Meisel, J. Phys. Chem., 1982, 86, 3391.

24 N. E. Cant, K. Critchley, H. L. Zhang and S. D. Evans, Thin Solid Films, 2003, 426, 31.

25 R. D. Badley, W. T. Ford, F. J. McEnroe and R. A. Assink, Langmuir, 1990, 6, 792.
26 Y. Sun, B. Mayers and Y. Xia, Nano Lett., 2003, 3, 675.

27 P. Hildebrandt and M. Stockburger, J. Phys. Chem., 1984, 88, 5935.

28 B. Pettinger, K. Krischer and G. Ertl, Chem. Phys. Lett., 1988, 151, 151.

29 H. Wetzel, H. Gerischer and B. Pettinger, Chem. Phys. Lett., 1981, 78, 392.

30 F. R. Dollish, W. G. Fateley and F. F. Bentley, Characteristic Raman frequencies of organic compounds, Wiley, New York, USA, 1974.

31 V. Amendola and M. Meneghetti, Adv. Funct. Mater., 2012, 22, 353-360.

32 Z. Movasaghi, S. Rehman and I. U. Rehman, Appl. Spectrosc. Rev., 2007, 42, 493.

33 J. C. Weaver, J. Cell. Biochem., 1993, 51, 426.

34 A. Huefner, W.-L. Kuan, R. A. Barker and S. Mahajan, Nano Lett., 2013, 13, 2463-2470.

35 C. Krafft, L. Neudert, T. Simat and R. Salzer, Spectrochim. Acta, Part A, 2005, 61, 1529.

36 S. K. Kim, T. H. Joo, S. W. Suh and M. S. Kim, J. Raman Spectrosc., 1986, 17, 381.

37 K. Kneipp, H. Kneipp, V. B. Kartha, R. Manoharan, G. Deinum, I. Itzkan, R. R. Dasari and M. S. Feld, Phys. Rev. E: Stat. Phys., Plasmas, Fluids, Relat. Interdiscip. Top., 1998, 57, 6281.

38 Y. Maruyama, M. Ishikawa and M. Futamata, Chem. Lett., 2001, 834.

39 A. Barhoumi, D. Zhang, F. Tam and N. J. Halas, J. Am. Chem. Soc., 2008, 130, 5523.

40 Y. S. Huang, T. Karashima, M. Yamamoto and H. Hamaguchi, Biochemistry, 2005, 44, 10009.

41 C. Matthaus, T. Chernenko, J. A. Newmark, C. M. Warner and M. Diem, J. Biophys., 2007, 93, 668.

42 E. A. Vitol, Z. Orynbayeva, G. Friedman and Y. Gogotsi, J. Raman Spectrosc., 2012, 43, 817.

43 A. Barhoumi, D. M. Zhang and N. J. Halas, J. Am. Chem. Soc., 2008, 130, 14040.

44 E. Le Ru and P. Etchegoin, Principles of Surface Enhanced Raman Spectroscopy and related plasmonic effects, Elsevier, Amsterdam, Netherlands, 1st edn, 2009. 